\title{
Multifunctional Coatings to Simultaneously Promote Osseointegration and Prevent Infection of Orthopaedic Implants
}

\author{
Jordan Raphel ${ }^{1}$, Mark Holodniy ${ }^{2}$, Stuart B. Goodman ${ }^{3}$, Sarah C. Heilshorn ${ }^{1} *$ \\ ${ }^{1}$ Department of Materials Science and Engineering, Stanford University, Stanford, CA \\ ${ }^{2}$ Division of Infectious Diseases, Palo Alto Veterans Affairs Health Care System, Palo \\ Alto, CA \\ ${ }^{3}$ Department of Orthopaedic Surgery, Stanford University, Stanford, CA
}

Prof. S. Heilshorn

476 Lomita Mall

McCullough Rm 246

Stanford, CA

94305, USA

heilshorn@stanford.edu

fax: 650-498-5596

Keywords: orthopaedic implants, functional coatings, osseointegration, antimicrobial treatments

\begin{abstract}
The two leading causes of failure for joint arthroplasty prostheses are aseptic loosening and periprosthetic joint infection. With the number of primary and revision joint replacement surgeries on the rise, strategies to mitigate these failure modes have become increasingly important. Much of the recent work in this field has focused on the design of coatings either to prevent infection while ignoring bone mineralization or vice versa, to promote osseointegration while ignoring microbial susceptibility. However, both coating functions are required to achieve long-term success of the implant; therefore, these two modalities must be evaluated in parallel during the development of new orthopaedic coating strategies. In this review, we discuss recent progress and future directions for the design of multifunctional orthopaedic coatings that can inhibit microbial cells while still promoting osseointegration.
\end{abstract}




\section{Introduction}

Orthopaedic implant use for joint replacements has been on the rise, with significant increases still projected over the next 15 years. ${ }^{[1]}$ The majority of procedures are knee and hip replacements, with over 700,000 knee and 300,000 hip replacements done annually in the United States. ${ }^{[2]}$ While these surgeries have a track record of decades of positive outcomes, approximately $10 \%$ of these implants fail prematurely, within the first 10-20 years, thereby affecting many tens of thousands of patients annually. ${ }^{[3]}$ Furthermore, as the US population continues to age and as life expectancy continues to increase, premature failures are not the only concern; many patients are now outliving their implants. This combination of factors leads to projections of a dramatic increase in implant failures in the near future.

The two leading causes of implant failure are aseptic loosening and infection. While the reported rates of these failures vary depending on the study, approximately $18 \%$ of implant failures are due to aseptic loosening while $20 \%$ of failures are attributed to infection. ${ }^{[4,5]}$ Additionally, these issues become even more prevalent in revised total joint arthroplasties. Aseptic loosening can originate from a variety of sources. These include micromotion of the implant relative to the bone during loading, the generation of implant wear particles that lead to inflammation and bone resorption, and poor osseointegration - the functional interface between the implant and the patient's bone. ${ }^{[6]}$ Implant site infections occur as microbes, particularly bacteria, become sessile and adhere to implant surfaces. These solid interfaces provide surfaces for bacterial attachment, proliferation, and biofilm formation, in which the adherent bacteria produce a protective, polymeric, extracellular substance, rendering these bacteria substantially more difficult to eradicate than individual suspended planktonic bacteria floating around the body. ${ }^{[7,8]} \mathrm{A}$ wide variety of bacteria can infect an implant, but a small subset of species makes up the majority of pathogens. Staphylococcus bacteria, most prominently S. aureus and S. epidermidis, account for close to $70 \%$ of orthopaedic implant infections, while Pseudomonas aeruginosa accounts for another $8 \%$ of infections. ${ }^{[9]}$

Aseptic loosening and implant infection appear to be mutually exclusive, particularly given the use of the word 'aseptic.' However, recent studies point to the potential connection between implants that have been reported to fail aseptically and 
latent occult infections that may have been missed prior to the time of diagnosis. ${ }^{[10]}$ Therefore, even in cases of implant failure where infection was not the primary cause, microbial presence may still play a critical role in initiating or accelerating the failure pathway.

Independently, the problems of aseptic loosening and infection are pressing for the orthopaedics field, and many excellent review articles cover the fields of osseointegration and infection prevention individually. ${ }^{[1-18]}$ However, the two issues are intimately related, as laid out by Gristina in his description of the "race for the surface"; if the host's cells can reach and occupy the implant surface first, not only will stronger tissue integration be achieved, but a defensive barrier will also be established against microbial attachment and colonization. ${ }^{[19]}$ Strong osseointegration and prevention of infection are both required for a successful implant, necessitating that implant designs consider both criteria simultaneously. In this review we describe several of the specific underlying mechanisms that lead to implant failure either by aseptic loosening or infection and potential design strategies to address these challenges (summarized in Table 1). In particular, with recent progress in understanding the connections between aseptic loosening and infection, this article will highlight recent works that address both problems in concert. 


\begin{tabular}{|c|c|}
\hline Causes of Implant Failure & Potential Solutions \\
\hline Gaps at prosthesis-bone interface & - Gap-bridging coatings \\
\hline Poor bone in-growth on implants & $\begin{array}{l}\text { - Calcium phosphate coatings } \\
\text { - Engineering surface topography } \\
\text { - } \quad \text { Bioactive glass coatings } \\
\text { - } \quad \text { 3D-printed coatings }\end{array}$ \\
\hline Poor bone deposition on implant surfaces & 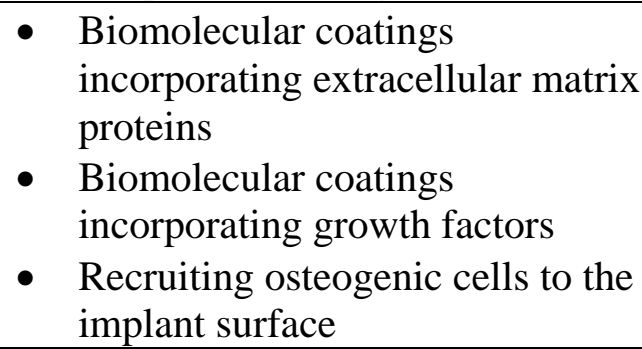 \\
\hline Initial microbial adhesion and infection & $\begin{array}{l}\text { - } \begin{array}{l}\text { Engineering surfaces to inhibit } \\
\text { bacterial adhesion }\end{array} \\
\text { - } \quad \text { Engineering bactericidal surfaces }\end{array}$ \\
\hline Late-stage infection & $\begin{array}{l}\text { - } \begin{array}{l}\text { Long-term presentation of } \\
\text { antibiotics }\end{array} \\
\text { - Slow-releasing antimicrobial } \\
\text { coatings } \\
\text { - Coatings containing antimicrobial } \\
\text { peptides }\end{array}$ \\
\hline Infection leading to osteolysis & $\begin{array}{l}\text { - Treatments to block inflammation } \\
\text { and differentiation signaling } \\
\text { cascades } \\
\text { - Immunomodulatory treatments }\end{array}$ \\
\hline
\end{tabular}

Table 1: Causes of orthopaedic implant failure and potential solutions to mitigate each cause and improve implant efficacy and lifetime.

\section{Challenges and Potential Solutions for Osseointegration}

Implant osseointegration relies on two distinct requirements. The first is obtaining initial implant stability during surgery, which then lays the groundwork for subsequent osseointegration of the implant as the patient heals. Ensuring implant stability is largely the responsibility of the surgeon and her/his team. Even technological solutions to improve initial implant fit, including automated imaging and robotic arm assistance platforms only assist, rather than replace, the surgery team. The second requirement is the prevention of later-stage loosening of the implant, which can be caused by a variety factors. These include lack of bone in-growth during healing, implant micromotion relative to the bone, adverse bone remodeling around the implant, and the formation of 
implant wear particles. This section will further investigate the challenges that can lead to later-stage implant loosening, technologies that have been explored to mitigate these factors or may be considered as the field progresses, and the successes of these technologies at simultaneously limiting infection.

\subsection{Challenge: Gaps at Prosthesis-Bone Interface}

A primary cause of aseptic loosening of joint replacement devices is implant micromotion due to gaps at the prosthesis-tissue interface. ${ }^{[6,20]}$ Increasing bone-implant contact reduces the size and number of gaps surrounding the implant, stabilizing the joint replacement prosthesis and limiting micromotion. As orthopaedic joint replacements are load-bearing implants, small micromotion may worsen over time with implant use, which can further progress towards greater micromotion, eventually leading to implant failure. ${ }^{[21]}$ Particularly large gaps are slow to be filled by bony in-growth during osteogenesis, further emphasizing the importance of initially limiting and eliminating the gaps. Gaps at the bone-implant interface can also lead to aseptic loosening in combination with implant debris, such as polymeric or metallic wear particles. ${ }^{[2]}$ The gaps can serve as conduits for wear particles to flow along the length of the implant, building up at the interface, and inhibiting direct prosthesis-bone contact. ${ }^{[6]}$ Furthermore, wear particles cannot be easily phagocytosed by macrophages, leading the cells to adopt an activated inflammatory state, in which they secrete a series of cytokines. These cytokines, such as tumor necrosis factor $\alpha(\mathrm{TNF}-\alpha)$, can lead to the generation of osteoclasts and the local resorption of bone tissue, effectively forming and enlarging gaps at the prosthesis-tissue interface. ${ }^{[23]}$ Local inflammation may lead to an altered immunological state that makes the implant more susceptible to microbial colonization. Illustrations of the different aseptic failure modes are presented in Figure 1. The potential implant instability caused by these gaps can progress in severity to the point that an implant revision is required. 


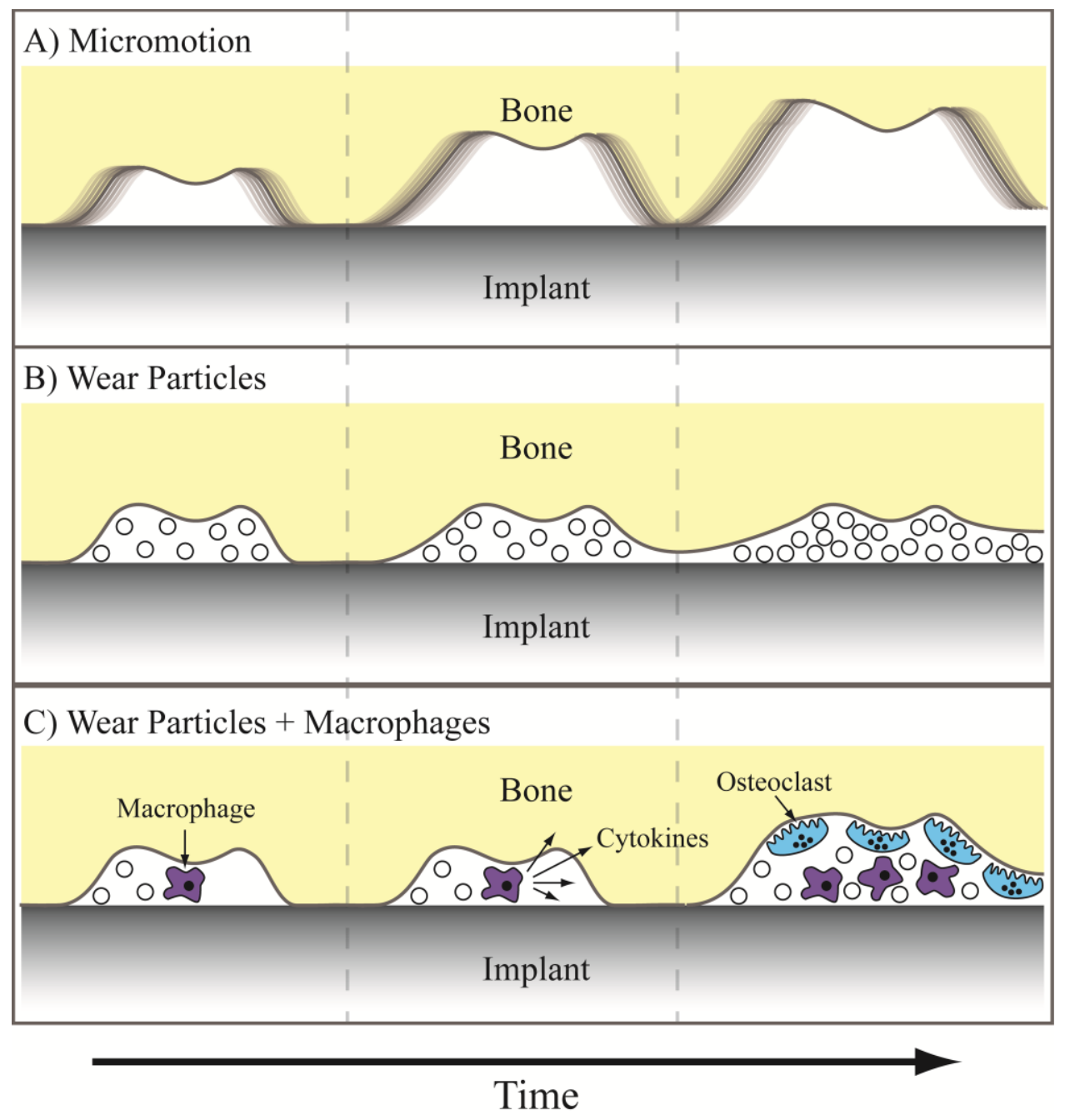

Figure 1: Aseptic loosening pathways associated with gaps at the bone-implant interface. A) Micromotion of the implant relative to the bone, leading to progressive worsening of micromotion. B) Wear particles interfering with direct contact between bone and implant. C) Wear particles activating macrophages, initiating a cytokine signaling cascade that leads to osteoclastogenesis and bone resorption.

\subsubsection{Potential Solution: Gap-Bridging Coatings}

One potential solution for gaps at the prosthesis-tissue interface is a coating that can swell or expand upon implantation in order to reduce micromotion and wear particleinduced osteolysis. Dynamic coatings can respond to small gaps on the micron scale around the implant in order to provide more direct contact between the implant and bone, thereby increasing the chance for a stable interface to form. A variety of different 
materials could be employed for these coatings, including hydrogels, foams, or deformable elastic metallic structures. Fournier et al. created a wire knit using nickel titanium (nitinol) for use as a flexible, gap-bridging implant coating. ${ }^{[24]}$ Thin nitinol wires were knit into a porous, superelastic structure that could be attached to the surface of an orthopaedic implant by a brazing process (Figure 2). In its expanded form, the knit coating was approximately $750 \mu \mathrm{m}$ thick, large enough to bridge large gaps surrounding an implant, but could be compacted down approximately $90 \%$ during implantation to provide conformal contact along the bone. Fournier et al. hypothesized the chronic outward force that the coating exerts on the surrounding bone may improve bone remodeling, thereby improving implant osseointegration. The pore size of the coating, which can be tuned by the knitting process, was $380 \mu \mathrm{m}$, which is within the size range shown to provide the strongest implant fixation. ${ }^{[25,26]}$ When compared to an implant with a titanium alloy (Ti6Al4V) plasma sprayed coating, the knit coating demonstrated more and deeper bone in-growth with similar amounts of inflammation and fibrous tissue formation. ${ }^{[24]}$ There is potential concern of using nitinol in implants due to its high nickel content and potential for the development of nickel sensitivity and release. The release of nickel ions and the stability of passivating oxides on NiTi surfaces is highly dependent on processing conditions, structure, and use, meaning the safety and biocompatibility of NiTi must still be evaluated on a case-by-case basis. ${ }^{[27]}$ Additionally, these superelastic porous coatings can only fill gaps where they are applied, necessitating that the coatings cover the entire implant surface in order to bridge all localized gaps. Though there are potential concerns about biocompatibility and gap filling along the entire implant surface, these coatings are amenable to additional engineering improvements that could increase their promise even further. Future improvements of the coating could include a filler material, such as a hydrogel, that could expand and compress within the knit coating, providing an even greater level of gap coverage to encourage bone in-growth. ${ }^{[28-30]}$ Such a hydrogel could also serve as a depot to deliver bioactive factors to promote osteogenesis and inhibit bacterial infection. ${ }^{[31,32]}$ Alternatively, much like drug-eluting stents, the nitinol wire could be coated with drugs, small molecules, or peptides that encourage osteogenesis and provide protection against bacterial infection. ${ }^{[33]}$ 

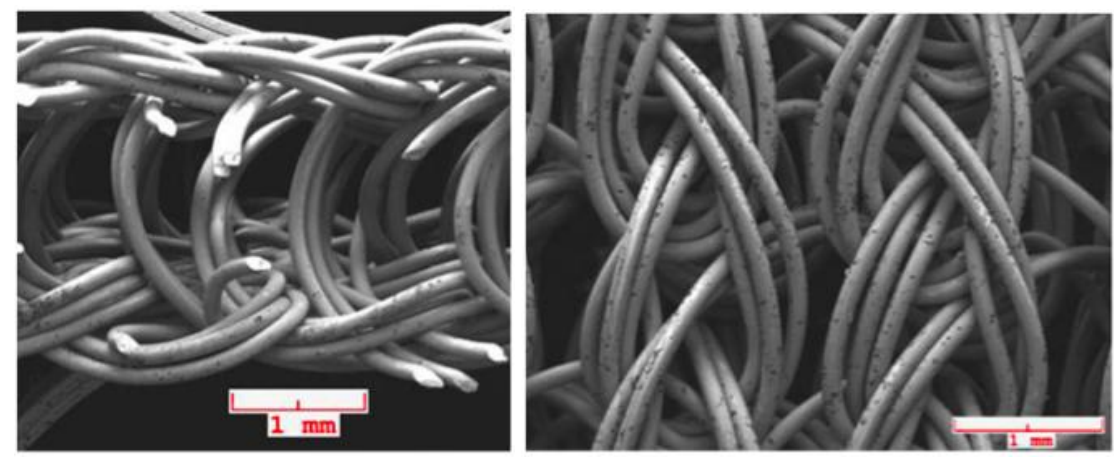

Figure 2: Knit nitinol wire fabric for gap-bridging coatings. Images show side (left) and top (right) faces of the knit coating. Source: Reprinted with permission from Springer, copyright $2014 .^{[24]}$

\subsection{Challenge: Poor Bone In-Growth on Implants}

Porous coatings on orthopaedic implants have been in use for roughly 40 years. ${ }^{[34]}$ The coatings, typically metallic or ceramic foams on the implant surface, are designed to facilitate bony in-growth ('osteoconduction') ${ }^{[35]}$ during the post-operative healing process to stabilize the implant. Many different types of porous structures have been developed, with beads commonly sintered together to form either open, interconnected pores or closed, individual pores. While these two porous structures are the most common, other examples include trabecular metals and wire meshes. ${ }^{[26]}$ However, despite their widespread use in clinical practice, premature revision rates have remained relatively high, around $10 \%$, for porous-coated joint prostheses, indicating their limited clinical success at improving osseointegration. ${ }^{[36,37]}$ As such, significant research has gone into optimizing the pore structure of the coatings, as well as identifying other techniques to encourage bony in-growth. Importantly, many of these technologies also affect the adhesion and viability of microbes on the implant surface, though specific mechanisms have not been well elucidated. These techniques can potentially be tuned to improve osteoconduction while inhibiting infection.

\subsubsection{Potential Solution: Calcium Phosphate Coatings}

Calcium phosphates (CaP), such as hydroxyapatite (HAp), are widely employed in bone tissue engineering for their osteoconductive properties. ${ }^{[38]}$ Recently, traditional calcium phosphate coatings have been combined with additives to create coatings that 
both improve antibacterial activity while maintaining strong osteoconductivity. Fielding et al. doped hydroxyapatite coatings with silver and strontium to increase infection defense and bone formation. ${ }^{[39]}$ The coatings allowed for osteoblast proliferation and improved alkaline phosphatase (ALP) production, indicating their ability to assist with osteogenesis. Furthermore, the coatings were highly antibacterial due to the release of silver ions, with activity expected to persist as silver release was still increasing after one week. Others have also doped silver into HAp coatings with similar results. ${ }^{[40]}$

Alternative calcium phosphate strategies combining the osteoconductive coating with bactericidal agents, such as antimicrobial peptides (AMP, discussed further in section 3.2.3.) also have been explored. In one example, AMPs were physically adsorbed onto the coatings to allow for temporal release. ${ }^{[41]}$ The AMP-CaP was adhesive to osteoblasts and, importantly, showed significantly improved bone-implant contact in a rabbit tibial model, providing in vivo evidence for better osseointegration compared with an uncoated titanium control. Furthermore, the AMP provided rapid and complete protection against both $S$. aureus and $P$. aeruginosa, killing all bacteria within 150 minutes. While this technology shows great promise, there was a significant burst release of the AMP, thus limiting the potential effective lifetime of the coating. Others incorporated the antibiotic norvancomycin into an HAp coating, which showed positive osteogenic and antibacterial abilities against $S$. aureus, but also suffered from a shortterm burst release. ${ }^{[42]}$ To slow down the release kinetics of the bactericides, degradable

polymer capping agents have been employed. ${ }^{[43]}$ While each example showcases the strong osteoconductive capabilities of calcium phosphate coatings and shows promise as an anti-infective, longer-term protection from bacterial infection is desirable for clinical use.

\subsubsection{Potential Solution: Engineering Surface Topography}

Another area of interest has been engineering of the micro- and nanostructure of the implant surface to improve interactions with osteogenic cells while simultaneously inhibiting bacterial adhesion. Increasing the surface area and porosity of the implant can improve bone in-growth and the coeffecient of friction between the bone and implant, 
thereby reducing micromotions and increasing osseointegration. For example, an anchorlike surface architecture was fabricated on implant surfaces using a direct metal laser sintering process. This anchor-like surface topography together with a secondary interconnected pore coating significantly improved primary implant fixation and bone ingrowth and decreased micromotion amplitude in both in vitro and large animal in vivo studies. ${ }^{[44,45]}$ Another method for altering the surface structure is to create nanopits through acid etching. Lan et al. combined acid etching with UV exposure to alter structure and surface energy. ${ }^{[46]}$ The resulting surface improved osteoblast ALP production and deposited mineralization while decreasing the presence of S. aureus and S. epidermidis by approximately $70 \%$. Etching has also been combined with techniques to alter microstructure, giving a hierarchically structured titanium surface. ${ }^{[47]}$ These surfaces showed improved in vivo mechanical stability and osseointegration in a rabbit tibial screw implantation model. The nano-micro-hierarchy provided defense against multiple Staphylococcus and Pseudomonas species over months, which is promising for addressing late-onset infections. ${ }^{[48]}$ One possible mechanism for this improved antimicrobial activity is an increase of surface free energy on the hierarchical surfaces, which was shown to alter the membrane structure of adhered bacteria, potentially inhibiting cellular activities. ${ }^{[47]}$ Developing a strategy that can maintain both the antimicrobial and osseointegrative capabilities long-term is critical for the viability of the implant technologies, making the early results for engineered surface topographies quite promising.

\subsubsection{Potential Solution: Bioactive Glass Coatings}

Bioactive glasses are synthetic, degradable ceramic materials containing key elements and molecules to encourage osteogenesis, and these materials have been in use for nearly 50 years. ${ }^{[49,50]}$ They have found continued use in the orthopaedics field due to their ability to deliver a surface apatite layer during dissolution, known as hydroxycarbonate apatite, which promotes osteogenesis. On their own, bioactive glasses have been shown to possess limited antimicrobial character by altering local $\mathrm{pH}$ during dissolution. However, when combined with additional components, bioactive glasses can 
both promote osteogenesis and provide significant antimicrobial activity. ${ }^{[49]}$ Ordikhani and Simchi created a composite implant coating by pairing bioactive glass with antibiotic-loaded chitosan. ${ }^{[51]}$ Chitosan, a popular coating material whose antimicrobial uses will be discussed in greater detail in section 3.1.2., was loaded with vancomycin and deposited in conjugation with bioactive glass nanoparticles onto titanium surfaces. The bioactive glass facilitated calcium phosphate mineral deposition and osteoblast adhesion. The antibiotic release rapidly inhibited $S$. aureus and continued over four weeks. Similar results were found using an ampicillin-loaded chitosan/bioactive glass hybrid to inhibit $S$. mutants. ${ }^{[52]}$

\subsubsection{Potential Solution: 3D-Printed Coatings}

Recent advances in 3D printing technology have improved its flexibility and precision to the point where it is now being extensively employed to create printed surfaces and coatings that may be utilized for orthopaedic applications. Though there is room for continued development and printer resolution to allow finer structures to be processed, 3D printing holds promise for creating customized coatings to improve the osseointegration of orthopaedic implants. ${ }^{[53]}$ One example of these coatings is 3D-printed trabecular titanium, described by Regis et al. ${ }^{[54]}$ The hexagonal, mesoporous structure was generated by specific electron beam melting, an additive manufacturing process. Trabecular titanium coatings enhanced osteogenic activity of human adipose-derived stem cells and osteoblasts in vitro and coated acetabullar cups showed strong osseointegration and survival rates in clinical studies. Additional examples of 3D-printed coatings and scaffolds utilizing calcium phosphate components have also been presented and show excellent promise for customizable implant surface engineering. ${ }^{[55-57]}$

\subsection{Challenge: Poor Bone Deposition on Implant Surfaces}

An ideal orthopaedic implant would not only allow for bony in-growth to secure the prosthesis, but would also encourage new bone deposition on its surface ('osteoinduction') ${ }^{[35]}$ to speed up the stabilization process and to provide a seamless bone-implant interface. Without osteoinductive functionality, implants can suffer from 
poor deposition of new bone tissue. Bony in-growth is required for successful stabilization, which can be compromised in patients with poor local bone quality, slow healing rates, and other confounding factors. Myriad surface coatings, additives, and bioactive cues have been incorporated with implants to render them osteoinductive, including growth factors and chemokines. There are additional operational concerns with these coatings, particularly around long-term storage, biomolecular activity, and adhesion strength. Still, given the roles of many of these biomolecules in native osteogenesis, they serve as attractive components for potential osteoinductive coating design.

\subsubsection{Potential Solution: Biomolecular Coatings Incorporating Extracellular Matrix Proteins}

Extracellular matrix (ECM) proteins have found use in bone tissue engineering applications in part because of their presence in physiological bone. Bone is a composite material containing both an inorganic mineral phase as well as an organic matrix phase ('osteoid'). Collagen I comprises approximately $90 \%$ of the osteoid phase, making it an obvious starting point for an osteoinductive biomolecular coating; however, this ECM is also known to be adhesive to multiple microbes. Engineered, collagen-mimetic peptide implant coatings have been used in place of human collagen I to limit bacterial adhesion and colonization while still providing osteoinductive cues. ${ }^{[58]}$ The collagen mimic was engineered to include specific ligand sequences that recognize highly expressed integrins on osteogenic cells. The coatings were adhesive for multiple osteogenic cell types with a similar level of adhesion as found on human collagen I coatings. Importantly, the collagen-mimetic coatings showed reductions in adhesion of S. aureus and S. epidermidis between 100-1,000 fold over human collagen I coatings, and 10-100 fold versus uncoated titanium. This collagen-mimetic protein serves as an excellent example of an osteoinductive biomolecular coating that also provides defense against implant infection.

Other groups utilized collagen coatings that release adsorbed antibiotics ${ }^{[59]}$ and silk coatings coupled with an anti-fouling surface ${ }^{[60]}$ as combinatorial protein coatings to improve osteoinduction. 


\subsubsection{Potential Solution: Biomolecular Coatings Incorporating Growth Factors}

One of the most widely used cues to initiate bone deposition is bone morphogenetic proteins (BMP), a family of potent osteogenic growth factors. ${ }^{[61]}$ In particular, BMP-2 has been used in conjunction with antimicrobial components to create osteoinductive coatings for orthopaedic implants. For example, the growth factor has been grafted to an anti-infective chitosan layer to create a multifunctional coating. ${ }^{[62]}$ Though the BMP-2 only mildly improved adhesion of osteogenic cells, it greatly improved both calcium mineral deposition and ALP activity compared to coatings lacking BMP-2. Furthermore, Shi et al. demonstrated that the grafted BMP-2 remained localized to the coating, implying that it could be osteoinductive long-term. In an alternative approach, BMP-2 was delivered as a soluble cue, using a biomolecular heparin carrier to control release. While this BMP-2 delivery strategy resulted in similar osteoblast adhesion, ALP activity, and mineral deposition results as the grafted BMP-2 study, it is expected that the osteoinductive potential would diminish over time as the BMP-2 was released from the coating. ${ }^{[63]}$

\subsubsection{Potential Solution: Recruiting Osteogenic Cells to the Implant Surface}

A newer strategy in the osteoinductive field is the recruitment of osteogenic cells through released signaling factors. Instead of solely relying on cells to passively find the osteoinductive coating, cells can be induced to "home" to the implant surface by creating a gradient of chemoattractive factors. One of the most well studied recruitment cues is the cytokine stromal cell-derived factor $1 \alpha(\mathrm{SDF}-1 \alpha)$, also known as CXCL12, which can mobilize multiple stem cell populations, including mesenchymal stem cells (MSCs) through their CXCR4 surface receptor. ${ }^{[64-67]}$ While a recruitment strategy might work independently, it could be most valuable when used in conjunction with localized osteogenic cues to encourage new bone deposition from the cells that get trafficked to the implant (Figure 3). For example, Zwingenberger, et al., found that delivery of SDF-1 $\alpha$

enhanced the efficacy of BMP-2 in generating new bone. ${ }^{[68]}$ The migration of MSCs to the site of a bone injury and bone volume were significantly improved using the cytokine combination at the injury site, resulting in substantially more bone regrowth in a rodent 
femoral defect model. Others have found similar results using the same combination of SDF-1 $\alpha$ and BMP-2, delivered either concurrently or sequentially. ${ }^{[69,70]}$ To the best of our knowledge, this strategy has not been used to improve the osseointegration of an orthopaedic implant, though its success in the literature suggests that it could be hugely beneficial, especially considering the local environment around orthopaedic implants. For instance, the stem portion of a hip prosthesis is implanted into the femoral medullary cavity, which contains a significant amount of bone marrow. Similarly, bone marrowderived MSCs are found in the metaphysis, where the posts of knee replacement prostheses are implanted. Additionally, SDF-1 $\alpha$ may also help prevent infection, as it is a chemoattractant for neutrophils. ${ }^{[71]}$ Summoning neutrophils to the implant site could limit the occurrence of infection through their ability to phagocytose microbes and recruit macrophages. 


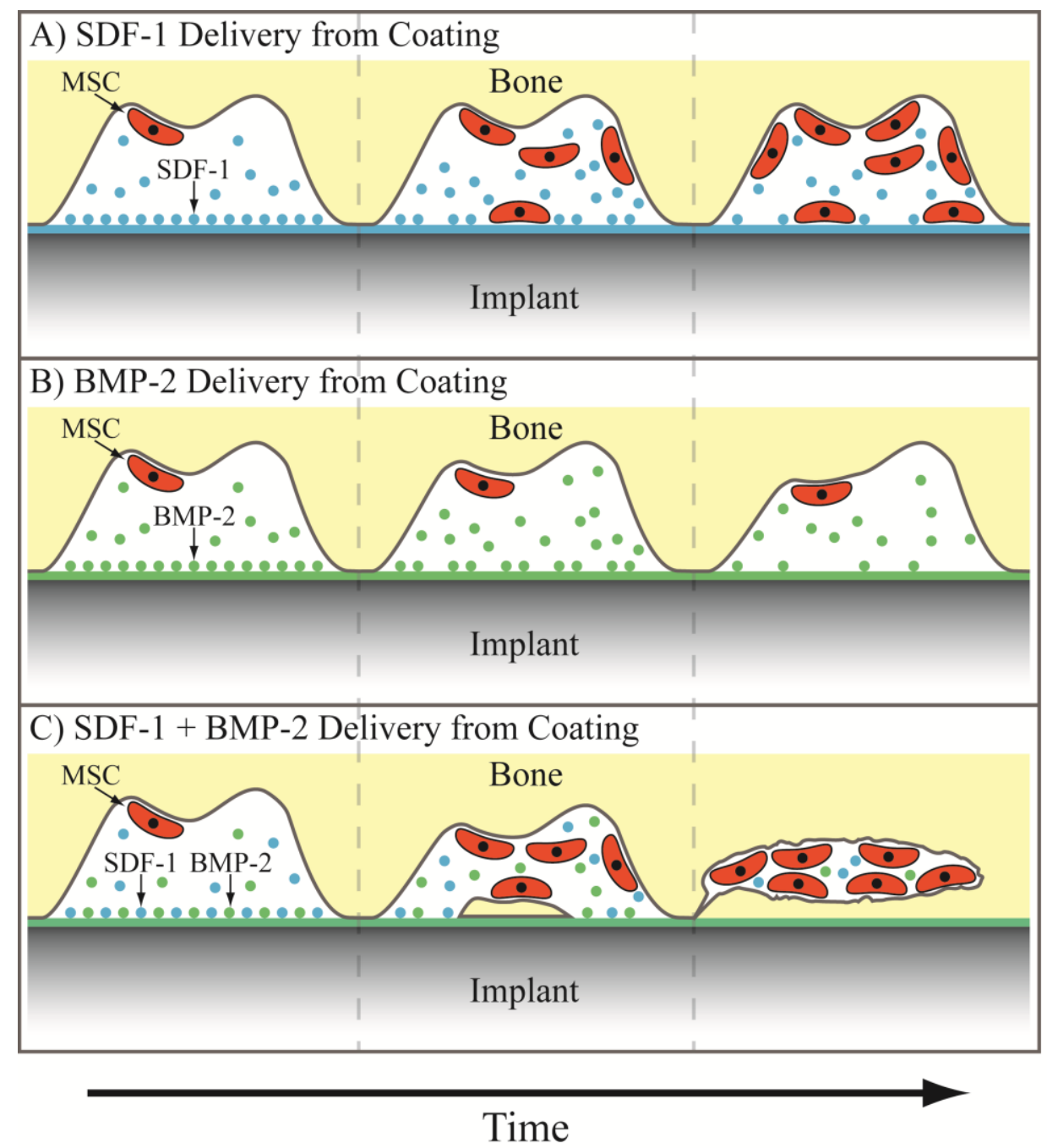

Figure 3: Potential strategy for improving osteogenesis using a combination of stem cell "homing" and osteogenic cues. A) Mesenchymal stem cell recruitment using chemoattractive gradient of SDF-1. B) Osteogenesis using BMP-2 to induce new bone formation from present mesenchymal stem cells. C) Combined strategy using both SDF-1 and BMP-2 to recruit mesenchymal stem cells and induce new bone formation concurrently.

\section{Challenges and Potential Solutions for Bacterial Infection of Implants}

Infection of orthopaedic implants can have a plethora of consequences, including hospitalization and costly revision surgery. ${ }^{[11]}$ Implant infection has been reported to be 
the new leading cause of orthopaedic implant removal, overtaking aseptic loosening. ${ }^{[4,72-}$ 74] Despite the biocompatibility of titanium and its alloys used for implants, bacteria easily and readily colonize these surfaces. Once bacteria adhere to the surface, they begin to proliferate, eventually reaching a high enough density to form a biofilm. The biofilm protects the bacterial colony with an extracellular polymeric substance, rendering it largely resistant to antibiotics, immune cells, and other potential infection defense mechanisms. ${ }^{[75]}$ Therefore, it is critical to address pathogenic bacteria as early as possible in order to minimize the chance of biofilm formation.

A myriad of strategies have been explored to limit microbial infections. The research can generally be broken down into addressing infections over two different time frames; techniques that can rapidly inhibit infection and techniques designed for longerterm defense. Both timescales are important from a clinical perspective; infections occurring within the first three months are defined as early while those manifesting after the initial period are defined as delayed and late. ${ }^{[76]}$ The first 6 hours after surgery are viewed as the particularly decisive period for preventing early infection, as the introduced pathogens have not yet begun rapid proliferation. ${ }^{[77]}$

Limiting the initial microbial adhesion to implant surfaces is critical to defend against early infection. During and for a few days after surgery, patients receive systemic antibiotics to address concerns of post-operative infection. However, many types of bacteria have developed resistance to various antibiotics, making alternative and redundant strategies attractive to further protect the patients. Strategies to limit bacterial adhesion are one way of addressing early infection. Another solution is bactericidal surfaces that can actively kill bacteria as they contact the implant surface. Within bactericidal techniques, some protect only the implant site, typically by directly coupling an antimicrobial agent to the surface, while others allow for the release of the antimicrobial agent, creating a zone of protection greater than just the implant surface but still much more localized than a systemic treatment. An entirely different strategy looks to stimulate and interact with the patient's own immune system. This immunomodulatory approach can either antagonize the immune system in a controlled manner to increase native defense against infection or down regulate an overactive defense that may be fighting infection but at the expense of implant function. 
Many antimicrobial strategies have been explored and reported in the literature. However, the evaluation of these strategies for orthopaedic implant applications is often limited only to their ability to prevent infections and does not consider their effects on osseointegration. Since both infection prevention and osseointegration are requirements for a successful implant, we will highlight antimicrobial strategies that also assess the impact on osseointegration, focusing specifically on multifunctional strategies.

\subsection{Challenge: Initial Microbial Adhesion and Infection}

The first phase of a deep implant infection is the initial adhesion of microbes at the site of implantation, beginning the process of infection-associated implant failure. To inhibit this first step, the implant surface can be rendered non-adhesive ('anti-fouling') or bactericidal. Each strategy has potential advantages and disadvantages. Anti-fouling coatings may be efficacious for longer periods of time because they are passive rather than active. However, many coatings that inhibit bacterial adhesion also limit mammalian

cell adhesion, which is desired for osteogenesis and implant fixation. ${ }^{[78]}$ Conversely, bactericidal surfaces not only inhibit the development of infection but can also kill the pathogen, decreasing the risk of a future infection. Nonetheless, even potent antimicrobial agents will rarely kill all local bacteria, leaving open the chance for a later infection or the development of resistance to the bactericide. Furthermore, anti-infective coatings will typically have a limited lifetime as the active agents are consumed or degraded over time, and the bactericide (and its potential degradation products) may have unknown, negative interactions with osteogenic cells. In this section, passive and active strategies to mitigate early-stage infection are highlighted.

\subsubsection{Potential Solution: Engineering Surfaces to Inhibit Bacterial Adhesion}

\section{Polymer Brushes}

Engineering surfaces to inhibit bacterial adhesion has been a consistent push within the biomedical industry. One technique commonly employed is creating an inert polymer brush layer on the substrate. The most widely studied polymer for this purpose is 
polyethylene glycol (PEG), which has been physically adsorbed, directly grafted, and grafted through intermediate bonding layers onto surfaces. ${ }^{[79]}$ In one example, an antifouling grafted PEG brush was modified with the cell-adhesive RGD ligand to regain interactions with osteoblasts. ${ }^{[80]}$ S. aureus adhesion was significantly reduced and, though Harris et al. did not directly assess osteoblast interactions, RGD-modified PEG has been shown to facilitate interactions with osteogenic cells. ${ }^{[81]}$

Other types of polymers have also been explored for anti-fouling surfaces, including synthetic and biopolymers. Dextran has been used in part due to its multiple sites for additional modification, such as tethering BMP-2 ${ }^{[82]}$ The anti-fouling nature of the dextran coating was confirmed, as adhesion of S. aureus and S. epidermidis were reduced by approximately 50\% compared to bare Ti6Al4V. Highlighted previously, Zhang et al. used a poly(methacrylic acid) (PMAA)-silk copolymer to inhibit S. aureus and S. epidermidis adhesion. ${ }^{[60]}$ The PMAA coating alone hindered adhesion of both bacteria and osteoblasts, demonstrating its anti-fouling functionality, but the inclusion of the grafted silk protein partially rescued osteoblast functionality.

Polymer brush coatings are passive solutions to create inert layers that limit protein adsorption and cell adhesion. However, uniform brush layers can be difficult to fabricate, and polymer brushes may degrade over time and are particularly susceptible to cleavage at the grafting point. ${ }^{[83]}$ Therefore, while polymer brushes can slow down the process of bacterial adhesion, they are unlikely to entirely eliminate infections on their own.

\section{Nanotube Coatings}

Titanium dioxide $\left(\mathrm{TiO}_{2}\right.$, 'titania') nanotube coatings are an interesting direction that has been pursued recently to limit bacterial adhesion. Additionally, titania nanotubes have been shown to have beneficial interactions with osteogenic cell types, making them a potentially useful tool for concurrently improving osseointegration. ${ }^{[84]}$ Das et al. synthesized titania nanotubes from a $\mathrm{Ti}$ surface using an anodization process in the presence of sodium fluoride and sulfuric acid. In order to provide antimicrobial efficacy, the nanotubes were then anodized with silver nitrate. ${ }^{[85]}$ While human osteoblast precursor cells adhered equally well to nanotube coatings with and without the silver 
nitrate coating, $P$. aeruginosa viability was decreased one thousand fold on the silver coated nanotubes. Subsequent research on titania nanotubes has yielded additional promising results. Peng et al. applied a simple nanotube array to Ti substrates and measured the osteogenic and antibacterial properties of the coating. ${ }^{[86]}$ The presence of the nanotubes improved the adhesion of osteogenic cells, with better adhesion on nanotubes of smaller diameter. Interestingly, S. epidermidis adhesion followed an opposite trend, with smaller tubes providing better inhibition. The results imply that nanotube optimization may be used to generate an osteogenic and anti-infective coating. A similar system was introduced by Izquierdo-Barba et al., who created titania nanocolumns on Ti6Al4V surfaces oriented approximately normal to the substrate. ${ }^{[87]}$ The densely packed columns had only a minimal effect on attachment of osteoblasts, but were able to significantly reduce the percent surface coverage and biofilm formation of multiple clinical $S$. aureus strains.

Nanotubes can also be modified to provide alternative antimicrobial and osteogenic mechanisms. For instance, zinc $(\mathrm{Zn})$ is required for multiple steps of bone formation, and its ions have been found to be bactericidal. Therefore, zinc was loaded onto a titania nanotube surface coating to simultaneously enhance osseointegration and prevent infection (Figure 4). ${ }^{[88]}$ Along with improvements in the in vitro osteogenic markers of ALP activation, mineral deposition, and enhanced mRNA expression levels of collagen I, osteocalcin, and osteoprotegerin; the Zn-loaded nanotubes significantly increased osseointegration in vivo in a rodent tibial insert model. Additionally, the $\mathrm{Zn}$ inhibited $S$. aureus growth, with longer-term bactericidal activity expected given the slow release of ions over one month. Other modification strategies have included the incorporation of silver ${ }^{[89]}$ and copper ${ }^{[90]}$ into nanotubes to serve as bactericidal agents, again taking advantage of the high surface area and loading capacity of nanotubes.
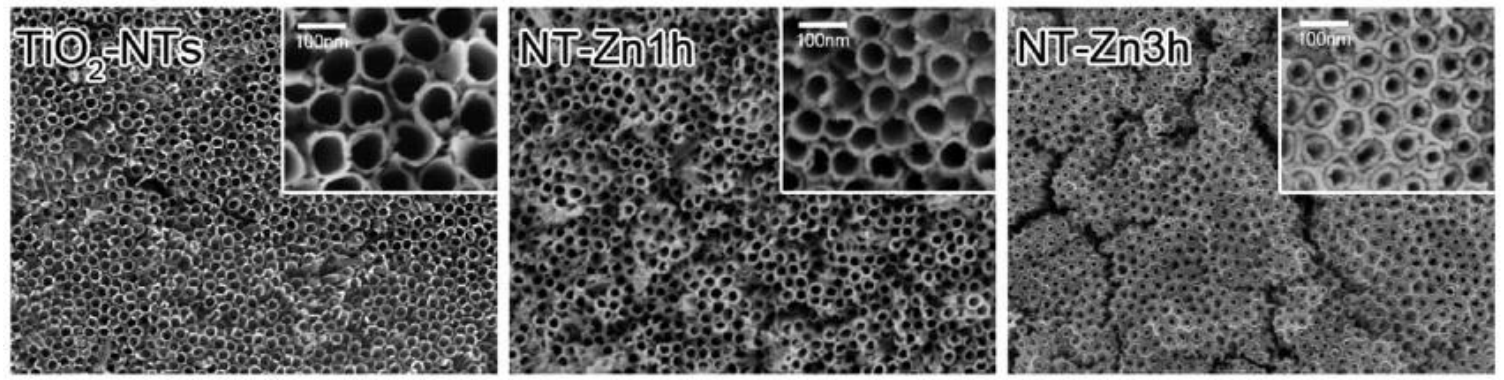
Figure 4: Scanning electron micrographs of titania nanotubes loaded with increasing amounts of zinc. Images show top-down views of nanotubes without exposure to zinc (left), and with increasingly long exposure to zinc solution of 1 hour (middle) and 3 hours (right). Source: Reprinted with permission from John Wiley and Sons, copyright 2013. ${ }^{[8]}$

\subsubsection{Potential Solution: Engineering Bactericidal Surfaces}

Multiple approaches have been used to kill bacteria on contact. One advantage of bactericides compared to anti-fouling coatings is the elimination of the pathogen; whereas anti-fouling coatings may allow the bacteria to return, bactericidal coatings can actually eradicate the root cause of infection. However, in order to entirely eliminate the risk of future infection, all of the pathogenic microbes would need to be purged, which is still unlikely using today's strategies. Instead, bactericidal coatings can simply mitigate the immediate risk of infection, and may eliminate the risk of infection altogether in conjunction with a healthy immune system. The most common bactericidal agent currently in use, both clinically and in research, is silver.

\section{Silver Implantation}

Silver has long been known to possess broad spectrum antimicrobial activity, and hence has been widely employed to fight infections within the medical device field. ${ }^{[91]} \mathrm{A}$ common strategy to introduce antimicrobial character to orthopaedic materials without significantly altering or coating the implant surface is through silver implantation, specifically silver plasma immersion ion implantation (Ag-PIII). ${ }^{[92-95]}$ Ag-PIII antimicrobial surfaces have been shown to have bactericidal efficacy against relevant bacterial species and to promote osteogenesis both in vitro and in vivo. The Ag-PIII process creates and embeds silver nanoparticles in substrates to ensure their long-term localization. The surfaces have been proven to be bactericidal, as opposed to anti-fouling, by collecting adherent bacteria and growing them in fresh culture, where a $99 \%$ reduction

in viability for S. aureus was observed. ${ }^{[94]}$ Meanwhile, the Ag-PIII surfaces showed good cytocompatibility with osteoblasts and facilitated higher bone volume, bone mineral density, and trabecular thickness than non-implanted surfaces in a dog model. ${ }^{[95]}$ The 
results further suggested that an optimal silver concentration exists, and exceeding that amount can be detrimental to bone growth, emphasizing the interplay between antimicrobial functionality and osseointegration. Ag-PIII has also been combined with other elemental implantation, including magnesium, zinc, and calcium, for additional antibacterial and osteogenic efficacy. ${ }^{[93,96,97]}$

\section{Chitosan Coatings}

An interesting bactericidal alternative to silver is the biopolymer chitosan. Chitosan is a cationic, linear polysaccharide derived from chitin, an abundant biopolymer in insect and crustacean exoskeletons. Chitosan has been widely studied in both bone tissue engineering and antimicrobial applications. Multiple reviews of the properties and uses of chitosan exist in the literature. ${ }^{[98-101]}$ Typically, chitosan is combined with osteogenic agents, such as RGD ligands, to create hybrid materials for orthopaedic implant applications. ${ }^{[102]}$ RGD-modified chitosan was found to still have the ability to decrease $S$. aureus and S. epidermidis adhesion by $67 \%$ and $85 \%$, respectively, while the RGD ligand improved expression of osteogenic markers. Chua et al. increased the level of complexity of the chitosan-RGD coating, creating layer-by-layer coatings of chitosan and hyaluronic acid with RGD ligands presented at the surface. ${ }^{[103]}$ These coatings also reduced bacterial adhesion independent of the presence of RGD, with $S$. aureus adhesion reduced by $75 \%$. Chitosan has also been complexed with BMP-2, yielding similarly promising results. ${ }^{[104]}$

Chitosan has also been combined with other antimicrobial agents to increase its bactericidal efficacy. For example, it has been used as a drug-carrier coating, loaded with adsorbed vancomycin. ${ }^{[105]}$ Chitosan nanoparticles have also been created and similarly loaded with the antibiotic ciprofloxacin. ${ }^{[106]}$ The nanoparticles exhibited a burst release of antibiotic and were able to reduce $S$. aureus load 20-fold over two days. However, the chitosan nanoparticles themselves were not able to hinder growth of multiple clinical $S$. aureus strains. It is possible that chitosan is a less effective antimicrobial agent in nanoparticle form and that it may require higher surface coverage to be inhibitory. Chitosan has also been employed as a conjugation vehicle for lauric acid, which also has

antimicrobial properties. ${ }^{[107]}$ Not only did the composite inhibit initial bacterial growth, 
but it also showed antibacterial efficacy over the course of one week, with greater than 95\% and $93 \%$ efficacy against $S$. aureus and $P$. aeruginosa, respectively. The ability of chitosan to both inhibit bacterial adhesion itself and to concurrently serve as a drug carrier in vitro makes it a very intriguing material to consider for future use, though drug release kinetics would ideally be tuned for slower, longer-term delivery.

\subsection{Challenge: Long-Term Prevention of Infection}

Orthopaedic implants are designed to have a functional lifetime of 20 years in the patient and are at risk to become infected during that entire time span. While infection due to virulent organisms, like $S$. aureus, typically manifests in the early stage, less virulent organisms can lead to chronic infections in the delayed and late stages. ${ }^{[108]}$ Therefore, developing technology to provide defense against infection over the course of weeks, months, and preferably years, is critical to reduce the overall rate of implant infection. Systemic antibiotics can still be administered for late infections, but often times the bacterial pathogens have formed biofilms, which protect the bacteria from antibiotics

and the immune system. ${ }^{[109-112]}$ Increasing the dose or duration of a systemic antibiotic may be effective, but host toxicity concerns become paramount. Additionally, different antibiotics that have better efficacy against biofilms, such as rifampin or daptomycin, may be used. However, antibiotic resistance in bacteria continues to increase, with the overuse of systemic antibiotics contributing to this problem. Development of resistance could still be an issue with local antibiotic presentation, but the risk is presumably decreased as fewer bacteria are interacting with the drugs. An ideal technology would provide both potent short-term as well as long-term, localized defense against infection while still promoting osseointegration.

\subsubsection{Potential Solution: Long-Term Presentation of Antibiotics}

A newer approach to the more traditional systemic antibiotic administration is localized antibiotic delivery. The drugs can be loaded into carriers that are attached to the implant itself, followed by subsequent passive drug release. Alternatively, antibiotics can be immobilized on the implant surface, a strategy whose success depends on the ability of 
the antibiotic to remain functional in its tethered form. Hickock and Shapiro described a method to covalently tether antibiotics such as vancomycin to implant surfaces including Ti and Ti6A14V. These substrates demonstrated bactericidal success against multiple types of bacteria, including S. aureus and S. epidermidis, over extended periods of time approaching one year and with multiple bacterial insults. ${ }^{[113-115]}$ Furthermore, vancomycin-functionalized $\mathrm{Ti}$ rods were found to reduce osteolysis in an infected rat femur implant model, demonstrating the efficacy of the tethered antibiotic in vivo.

\subsubsection{Potential Solution: Slow-Releasing Antimicrobial Coatings}

The continual increase of bacterial antibiotic resistance has pushed alternative antimicrobial treatments into the spotlight. The most common strategy is the release of silver ions, though other ions have also been shown to possess bactericidal efficacy. Similar to antibiotic strategies, long-term defense against infection is key to protecting the implants for as much of their lifetime as possible, thus necessitating an understanding of the release rate of the ions over time. This characteristic separates this section from section 3.1.2., where significant release of silver was not the primary mode of action (Figure 5).
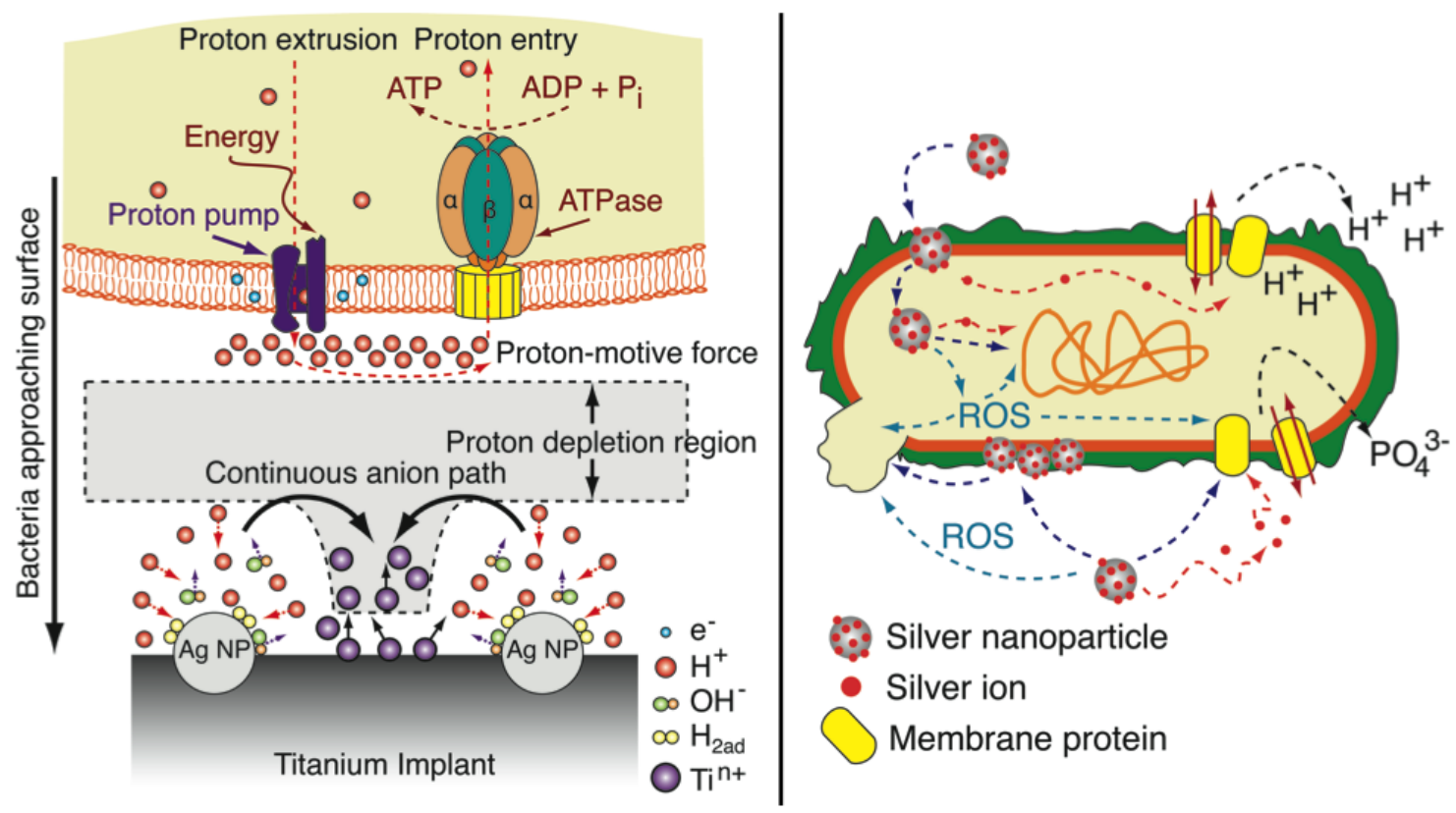
Figure 5: Proposed bactericidal mechanisms of embedded and released silver. Microgalvanic interactions of embedded silver nanoparticles lead to the generation of protons for applications where ion generation is slow (left). Free silver ions and nanoparticles interact with membranes, transport proteins, and DNA to compromise bacteria (right). Source: Adapted and reproduced with permission from Elsevier, copyright 2011 (left) and Springer, copyright 2010 (right). ${ }^{[92,116]}$

\section{Silver Release}

Silver is an attractive bactericide in part because of its broad-spectrum efficacy, as it has been shown to kill both Gram-positive and Gram-negative bacteria. ${ }^{[116]}$ It has also been shown to be cytocompatible in a variety of studies, such as those previously discussed, though it can still be toxic to mammalian cells at higher concentrations. ${ }^{[117]}$ Silver ions are frequently released from the surfaces of silver nanoparticles due to their high surface to volume ratios. Liu et al. embedded pure silver nanoparticles into a poly(lactide-co-glycolide) (PLGA) coating which widely inhibited Gram-positive and Gram-negative bacteria at multiple inoculum concentrations and times in a concentration dependent manner, while also improving ALP activation, mineral deposition, and expression of osteogenic markers (Figure 6). ${ }^{[118]}$ In vivo, osteolysis was found around implants without silver nanoparticles present, while bone formation was seen around coatings containing the silver in a rodent femoral implant model. After 8 weeks, no bacteria and minimal inflammatory cells were found in the tissue surrounding the silvercontaining implant, while both bacteria and inflammatory cells were present in the absence of silver, demonstrating the anti-infective ability of the silver over time. Others have loaded silver into titania nanotubes ${ }^{[119]}$, layer-by-layer polymeric coatings ${ }^{[120]}$, and self-assembled monolayers ${ }^{[121]}$ to demonstrate significant bactericidal efficacy. 


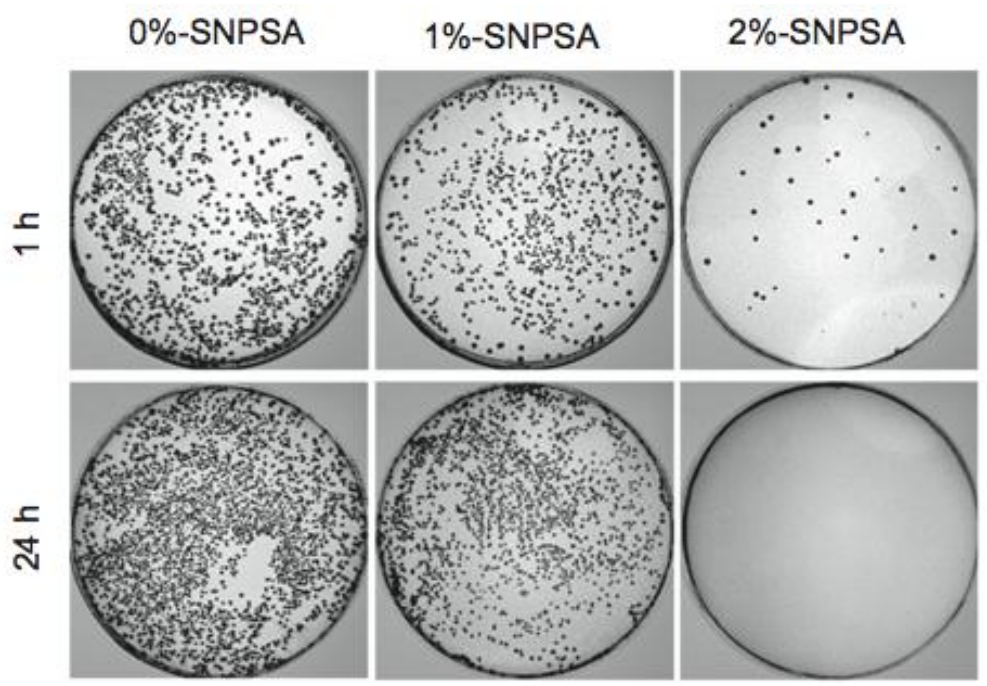

Figure 6: S. aureus growth after exposure to silver nanoparticle coatings. Increasing amounts of silver were used from left to right, with bacterial exposure times of 1 and 24 hours. After exposure, bacteria adherent to the coating surfaces were detached and plated. SNPSA: silver nanoparticle/PLGA-coated stainless steel alloy. Source: Reprinted with permission from Elsevier, copyright 2012. ${ }^{[18]}$

\section{Alternatives to Silver}

Much of the public concern over the development of bacterial resistance has been focused on antibiotics. However, there has been recent evidence of clinical bacterial isolates possessing plasmids containing sil genes known to confer resistance to silver, which could have major implications for its use as a bactericide in wound dressings and medical device coatings, including in orthopaedic applications. ${ }^{[122]}$ As such, antimicrobial alternatives to silver ions have been receiving more attention. Some technologies combine silver with other agents, while others entirely pass over silver as an option, citing concerns over cytocompatibility and future spread of silver resistance. For example, zinc incorporated into titania nanotubes was slowly released over the course of weeks, nearly completely eliminated $S$. aureus and E. coli bacteria, and promoted differentiation of osteogenic cells. ${ }^{[123]}$ Zinc has been employed in other nanotube arrays ${ }^{[124]}$, ion-implanted into $\mathrm{Ti}^{[125]}$, and incorporated into $\mathrm{TiO} 2$ coatings ${ }^{[126]}$ with varying antimicrobial efficacy, release profiles, and osteogenic potential. It seems possible, if not likely, that resistance to zinc will also develop over time. However, since its use as a bactericidal agent is recent, the hope is that resistance will not develop 
quickly. Still, one way of fighting resistant bacteria is to combine multiple agents together for broader defense.

Svensson et al. applied a nanostructured coating to titanium, containing silver, gold, and palladium, which had previously been used to render catheters antimicrobial. ${ }^{[127]}$ The coatings inhibited S. aureus adhesion to the implant while not interfering with in vivo measures of osseointegration - new bone formation, total bone area, and bone-implant contact area. The combination of multiple bactericidal agents may not only produce a strong defense against infection, but could slow down the development of bacterial resistance. A combination of zinc and silver nanoparticles was used to compromise both $S$. aureus and E. coli on the implant surface through direct microgalvanic interactions with the silver nanoparticles. These same nanoparticles were also found to be active in a rat femoral model against planktonic pathogens in the local region of the implant through interactions with leached zinc ions. ${ }^{[96]}$ Again, by combining multiple strategies, a wider spectrum of potential infections can be addressed. Conceivably, the most potent strategy for creating an antimicrobial coating that would have long-term efficacy involves the combination of multiple bactericidal agents with varying acute and chronic release profiles and/or presentation modes.

\section{Triggered Release}

Antibiotics can also be released over time, such as from titania nanotube carriers. ${ }^{[128]}$ One issue with diffusive release of antibiotics is the difficulty in controlling the release profile, and these systems often suffer from an initial burst release. Burst release is suboptimal because it represents a rapid depletion of the antimicrobial agent and because the high local concentrations upon release may be cytotoxic to host cells. Active drug release triggered by the presence of pathogenic microbes would be an immense step towards the development of long-term microbial defense. This strategy could help reduce the development of antibiotic resistance, as doses would only be applied in the event of microbial insult. Furthermore, defense lifetime could be increased, since the drug reservoir would only be depleted at specific times, rather than continuously. Bioresponsive, 'smart' carriers have been utilized in other biomedical applications $^{[129-131]}$ but are just beginning to be used for the selective delivery of 
antibiotics. For example, liposomes have been engineered to release an antibiotic payload in the presence of toxins secreted by S. aureus. ${ }^{[132]}$ Another potential solution would be utilizing multiple layers of passive release carriers, such as loaded nanoparticles or layerby-layer assemblies, capped with either a slowly degrading coating or an active release trigger, combining some of the previously outlined strategies. By using multiple layers, an initial burst release could be designed to protect the implant from immediate acute infections while the longer release portions would provide a chronic local defense.

\subsubsection{Potential Solution: Coatings Containing Antimicrobial Peptides}

Antimicrobial peptides (AMPs) represent a new class of antimicrobial agents that are derived from nature's various microbial defense mechanisms, particularly from organisms' innate immune defense. AMPs are typically short, amphiphilic, cationic peptides that can interfere with microbial membranes and, upon entry into the cell cytoplasm, intracellular targets. ${ }^{[133,134]}$ Since AMPs require a physical interaction with cell membranes, they can be active either floating free in solution or grafted onto a surface, assuming they possess enough conformational flexibility to properly reach and interact with their targets. For example, Kazemzadeh-Narbat et al. demonstrated that release of the AMPs Tet213 and HHC36 from calcium phosphate coatings had immense antimicrobial efficacy against $S$. aureus and $P$. aeruginosa within hours, killing virtually all bacteria. ${ }^{[41,135]}$ To achieve slower release kinetics, AMPs were then combined with titania nanotube-calcium phosphate layer-by-layer coatings with a phospholipid capping agent. ${ }^{[136]}$ With this strategy, the release lifetime was extended to several days, though further development to increase both the initial loading and release timeline would be desired to provide longer-term protection. AMPs have also been immobilized on hydrophilic polymer brushes, creating surfaces that are inhibitory to both $P$. aeruginosa viability and surface adhesion. ${ }^{[137]}$ With their mechanisms of action still being elucidated and a huge number of potential peptide sequences left to explore, the use of AMPs will likely greatly increase in the near future. Furthermore, given the current belief that AMPs physically interact with microbes, they may provide longer-term protection than other agents that become depleted over time. However, the AMPs themselves will be subject to 
proteolytic degradation. One proposed solution is engineering AMPs using the D-form of amino acids to inhibit protease attack. ${ }^{[134]}$ These enantiomer-substituted AMPs have been shown to maintain their bactericidal properties. ${ }^{[138-140]}$

\subsection{Challenge: Infection Leading to Osteolysis}

Throughout this article, the concepts of improved osseointegration and prevention of bacterial infection have been presented in parallel. However, they can have a direct relationship, particularly when infection leads to osteolysis. This process begins when macrophages recognize lipopolysaccharide (LPS, also known as endotoxin) within bacterial cell walls, causing the macrophages to become activated. ${ }^{[141]}$ Activated macrophages and many subsequent signaling molecules, including toll-like receptors, play significant roles in both infection-mediated osteolysis and aseptic loosening through osteoclastogenesis, highlighting the overlap between osseointegration and infection pathways. ${ }^{[142]}$

\subsubsection{Potential Solution: Treatments to Block Inflammation and Differentiation Signaling Cascades}

Macrophages home to sites of infection by detecting components of bacterial cells, such as LPS. ${ }^{[143]}$ Once at the infection site, the macrophages start their own signaling cascade, in combination with bone marrow stromal cells, which eventually leads to differentiation into osteoclasts and hence local bone resorption. ${ }^{[144]}$ Potential strategies to limit infection-mediated osteolysis and implant loosening would include the disruption of either the signaling that homes the macrophages to the implant site or macrophage differentiation into osteoclasts. The latter may be more beneficial for the patient overall, since having macrophages present will help address the underlying infection. Osteoclastogenesis requires signaling cues such as colony-stimulating factor-1 (CSF-1) and receptor activator of nuclear factor- $\mathrm{KB}$ ligand (RANKL). ${ }^{[144,145]}$ It has been shown that osteoclastogenesis can be mitigated in vivo by inhibiting the pathway leading to NF-kB production by use of a soluble protein. ${ }^{[146]}$ By incorporating a released or tethered inhibitor to an implant coating, similar inhibition of osteoclastogenesis may be 
achieved. Local and targeted delivery of osteoprotegerin or related peptides from a coating could also be used to block differentiation, as it has been successful at inhibiting osteoclastogenesis when delivered systemically. ${ }^{[147]}$ Additionally, hindering osteoclastogenesis could be done in a 'smart' fashion. Macrophages responding to infection secrete a variety of biomolecules, including proteases, which could be used as triggers for the release of an interfering peptide.

\subsubsection{Potential Solution: Immunomodulatory Treatments}

Much of the initial research on implantable biomaterials was focused on creating bioinert surfaces that would have minimal interaction with the host. However, there is currently significant interest in materials that can generate a consistent yet controlled level of immune response, termed 'immunomodulation'. In addition to speeding up tissue regeneration $^{[148]}$, immunomodulation can also be used to prime or trigger the body's innate immune system, and hence indirectly prevent infections. However, since chronic stimulation of the innate immune system can lead to sepsis ${ }^{[149]}$, it is important that immunomodulatory responses be carefully engineered and that the response remain localized. Still, the potential to stimulate the body's innate immune system to protect against infection, as opposed to attempting to directly interfere with the pathogenic microbes, would be very valuable in creating long-term, broad-spectrum defense. As a demonstration of this potential, LL-37, a cathelicidin peptide, was shown to have no specific antimicrobial functionality of its own against $S$. aureus, but was antimicrobial in vivo due to its immunomodulatory effects, protecting rodents from acquiring $S$. aureus and Salmonella infections. ${ }^{[150]}$ The anti-infective mechanism could be similar to that of the IDR-1 peptide, which has no independent antimicrobial character but is anti-infective due to its ability to stimulate factors within the innate immune system, including toll-like receptors, while controlling inflammatory response. ${ }^{[151]}$ There has been an increasing understanding of the role of AMPs in immunomodulatory activities, suggesting that they may be good candidates for these types of immune system-instructive treatments in addition to their direct bactericidal roles (Figure 7). ${ }^{[152]}$ Modifying orthopaedic implants with coatings that could present or release immunomodulatory agents could serve as an 
antimicrobial defense, providing both acute and chronic protection, thereby limiting the chances for infection leading to osteolysis and implant loosening.

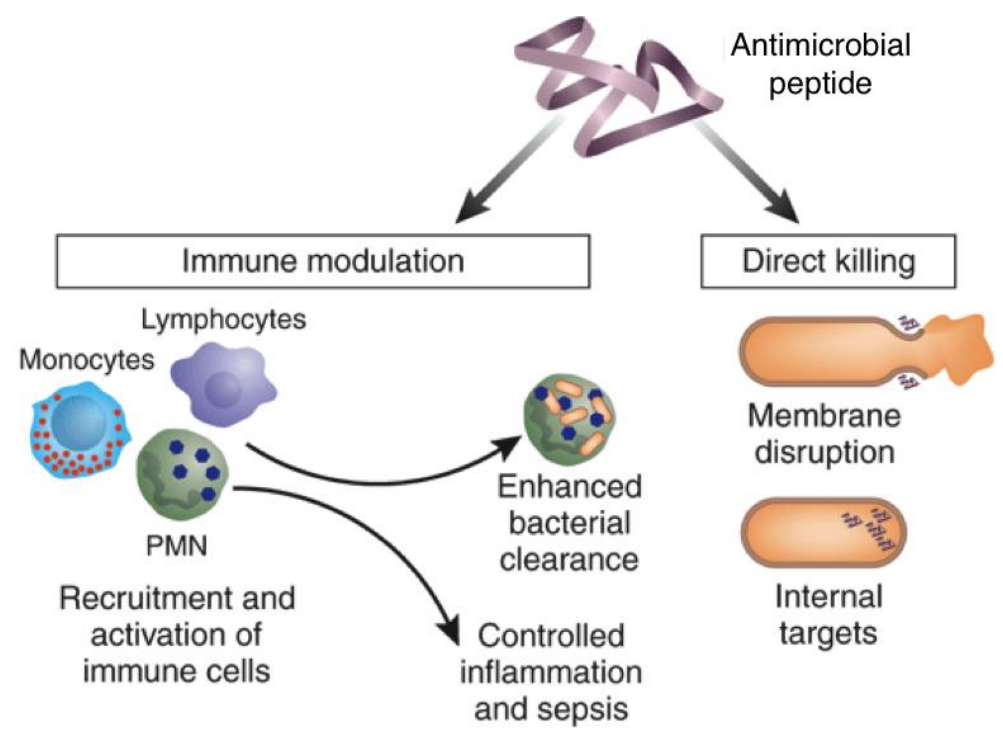

Figure 7: Schematic of the multiple possible modes of action for antimicrobial peptides (AMPs) in protecting against infection. AMPs can act as direct bactericides (right) or can serve as immunomodulatory agents (left). Source: Adapted and reprinted with permission from Nature Publishing Group, copyright 2006. ${ }^{[134]}$

\section{Future Direction}

Addressing the clinical challenges of aseptic loosening and implant infection have clearly drawn much attention from the research community. While significant advances have been made in coating technologies to reduce failure either by loosening or infection, the development of technologies to address both challenges simultaneously is ongoing. A clear future direction for the field is the development of multifunctional implant coatings that can effectively balance osseointegration and microbial challenges.

Since there is significant interaction between mammalian cells and microbes in situ, tailoring a solution to improve the adhesion and function of the former or hinder the adhesion and function of the latter may lead to unintended consequences. Ideally, an implant coating would only need to include a single design element that could simultaneously promote interactions with host tissue cells while inhibiting microbial interactions. However, until these specific design elements are identified, new implant 
coatings will likely require the combination of multiple functional elements in order to address both the aseptic loosening and the implant infection failure modes simultaneously. Myriad technologies that begin to address these failure modes in concert have been presented in this review. These can serve as the initial building blocks for future implant coatings, but none are yet sufficient on their own. Instead, combinations of these technologies will likely need to be exploited. For example, the idea of combining a gap-bridging elastic mesh with a hydrogel depot containing growth factors was postulated. Similarly, the long-term, anti-infective properties of titania nanotube coatings could potentially be improved by grafting immunomodulatory peptides to the coating surface, thereby generating coatings that protect against infection in both the short- and long-term.

Along with combining design elements to make multifunctional coatings, the ideal coating should also be cost-effective, applicable to any implant geometry, and maintain the shape and feel of the implants. The shifting focus in healthcare to better preventive care and fee-for-value models has placed increased scrutiny on costs and effectiveness of treatments. While an additional premium would certainly be warranted for a coating technology that increased implant efficacy and reduced the revision rate, the cost still needs to be balanced with the additional benefits in order to ensure widespread reimbursement from healthcare payers. The coatings should also be processable in a scalable and implant-agnostic manner to ensure extensive availability to patients. While the joint replacement prostheses market is fragmented among a handful of key manufacturers, each has exclusive implant geometries, sizes, recommended implantation procedures, and surfaces. Creating coatings that could be universally applied and effective on devices from any manufacturer would allow the most patients to benefit from the technology and have the greatest overall impact on reducing implant failures. Finally, the coatings should minimally affect the size, shape, and feel of the implants. By maintaining implant fidelity on the macroscopic level, the amount of additional surgeon training and experience needed to effectively utilize the coated implants will be minimized. This will speed up the adoption of the new coating technology without generating a steep learning curve, again providing the greatest benefit to patients. 


\section{Conclusions}

Orthopaedic implants are widely used and highly successful treatments for musculoskeletal issues. Their success can be undermined by poor osseointegration and infection, the two leading causes of implant failure and revision surgeries. A variety of strategies have been studied to improve osseointegration and to prevent infection, though typically proposed solutions have only addressed one of the two issues. In this review, we highlighted technologies that have the potential to address these issues in tandem to improve the overall function and lifetime of orthopaedic implants. Certain technologies, such as osteoconductive calcium phosphate and antimicrobial silver coatings, have a longer track record and are now being used in combination with other treatments. Others, such as gap-bridging coatings to reduce micromotion and immunomodulatory treatments to prime the innate immune system, are in their earliest stages but possess great potential for future success. The ideal orthopaedic implant coating will likely be multifunctional, combining different technologies to simultaneously promote osseointegration while inhibiting microbial infection. Our hope is that new understanding and new directions of research will result in patients receiving these ideal implants, thereby reducing premature implant failures and also extending overall implant lifetimes.

\section{Acknowledgments}

We acknowledge the Robert L. and Mary Ellenburg Professorship at Stanford University (SBG) and funding from the National Science Foundation (DMR 0846363 to SCH) and the National Institutes of Health (U19 AI116484-01, R21 EB018407-01, R21 AR062359-01 to SCH).

[1] S. Kurtz, K. Ong, E. Lau, F. Mowat, and M. Halpern, "Projections of primary and revision hip and knee arthroplasty in the United States from 2005 to 2030", J Bone Joint Surg Am, 2007, 89, 780.

[2] Cdc. National hospital discharge survey, Available from: http://www.cdc.gov/nchs/fastats/inpatient-surgery.htm accessed: August, 2015.

[3] S. Kurtz, F. Mowat, K. Ong, N. Chan, E. Lau, and M. Halpern, "Prevalence of primary and revision total hip and knee arthroplasty in the United States from 1990 through 2002", J Bone Joint Surg Am, 2005, 87, 1487. 
[4] K. J. Bozic, S. M. Kurtz, E. Lau, K. Ong, V. Chiu, T. P. Vail, et al., "The epidemiology of revision total knee arthroplasty in the United States", Clin Orthop Relat Res, 2010, 468, 45.

[5] K. J. Bozic, S. M. Kurtz, E. Lau, K. Ong, T. P. Vail, and D. J. Berry, "The epidemiology of revision total hip arthroplasty in the United States", J Bone Joint Surg Am, 2009, 91, 128.

[6] M. Sundfeldt, L. V. Carlsson, C. B. Johansson, P. Thomsen, and C. Gretzer, "Aseptic loosening, not only a question of wear: a review of different theories", Acta Orthop, 2006, 77, 177.

[7] J. W. Costerton, P. S. Stewart, and E. P. Greenberg, "Bacterial biofilms: a common cause of persistent infections", Science, 1999, 284, 1318.

[8] L. Hall-Stoodley, J. W. Costerton, and P. Stoodley, "Bacterial biofilms: from the natural environment to infectious diseases", Nat Rev Microbiol, 2004, 2, 95.

[9] D. Campoccia, L. Montanaro, and C. R. Arciola, "The significance of infection related to orthopedic devices and issues of antibiotic resistance", Biomaterials, 2006, 27, 2331.

[10] E. M. Greenfield, Y. Bi, A. A. Ragab, V. M. Goldberg, J. L. Nalepka, and J. M. Seabold, "Does endotoxin contribute to aseptic loosening of orthopedic implants?", J Biomed Mater Res B Appl Biomater, 2005, 72, 179.

[11] L. Zhao, P. K. Chu, Y. Zhang, and Z. Wu, "Antibacterial coatings on titanium implants", J Biomed Mater Res B Appl Biomater, 2009, 91, 470.

[12] D. Campoccia, L. Montanaro, and C. R. Arciola, "A review of the biomaterials technologies for infection-resistant surfaces", Biomaterials, 2013, 34, 8533.

[13] F. Costa, I. F. Carvalho, R. C. Montelaro, P. Gomes, and M. C. Martins, "Covalent immobilization of antimicrobial peptides (AMPs) onto biomaterial surfaces", Acta Biomater, 2011, 7, 1431.

[14] M. L. Knetsch, and L. H. Koole, "New strategies in the development of antimicrobial coatings: the example of increasing usage of silver and silver nanoparticles", Polymers, 2011, 3, 340.

[15] A. Simchi, E. Tamjid, F. Pishbin, and A. R. Boccaccini, "Recent progress in inorganic and composite coatings with bactericidal capability for orthopaedic applications", Nanomedicine, 2011, 7, 22.

[16] R. Branemark, P. I. Branemark, B. Rydevik, and R. R. Myers, "Osseointegration in skeletal reconstruction and rehabilitation: a review", J Rehabil Res Dev, 2001, 38, 175.

[17] S. B. Goodman, Z. Yao, M. Keeney, and F. Yang, "The future of biologic coatings for orthopaedic implants", Biomaterials, 2013, 34, 3174.

[18] A. Wennerberg, and T. Albrektsson, "Effects of titanium surface topography on bone integration: a systematic review", Clin Oral Implants Res, 2009, 20 Suppl 4, 172.

[19] A. G. Gristina, "Biomaterial-centered infection: microbial adhesion versus tissue integration", Science, 1987, 237, 1588.

[20] S. B. Goodman, "The effects of micromotion and particulate materials on tissue differentiation. Bone chamber studies in rabbits", Acta Orthop Scand Suppl, 1994, 258,1 . 
[21] E. R. Valstar, R. G. H. H. Nelissen, J. H. C. Reiber, and P. M. Rozing, "The use of Roentgen stereophotogrammetry to study micromotion of orthopaedic implants", ISPRS J Photogramm Remote Sens, 2002, 56, 376.

[22] T. P. Schmalzried, M. Jasty, and W. H. Harris, "Periprosthetic bone loss in total hip arthroplasty. Polyethylene wear debris and the concept of the effective joint space", J Bone Joint Surg Am, 1992, 74, 849.

[23] H. C. Amstutz, P. Campbell, N. Kossovsky, and I. C. Clarke, "Mechanism and clinical significance of wear debris-induced osteolysis", Clin Orthop Relat Res, $1992,7$.

[24] E. Fournier, R. Devaney, M. Palmer, J. Kramer, R. El Khaja, and M. Fonte, "Superelastic orthopedic implant coatings", J Mater Eng Perform, 2014, 23, 2464.

[25] J. D. Bobyn, R. M. Pilliar, H. U. Cameron, and G. C. Weatherly, "The optimum pore size for the fixation of porous-surfaced metal implants by the ingrowth of bone", Clin Orthop Relat Res, 1980, 263.

[26] G. Ryan, A. Pandit, and D. P. Apatsidis, "Fabrication methods of porous metals for use in orthopaedic applications", Biomaterials, 2006, 27, 2651.

[27] S. Shabalovskaya, J. Anderegg, and J. Van Humbeeck, "Critical overview of Nitinol surfaces and their modifications for medical applications", Acta Biomater, 2008, $4,447$.

[28] C. Von See, N. C. Gellrich, U. Jachmann, M. W. Laschke, K. H. Bormann, and M. Rucker, "Bone augmentation after soft-tissue expansion using hydrogel expanders: effects on microcirculation and osseointegration", Clin Oral Implants Res, 2010, 21, 842.

[29] S. Srouji, A. Rachmiel, I. Blumenfeld, and E. Livne, "Mandibular defect repair by TGF-beta and IGF-1 released from a biodegradable osteoconductive hydrogel", $J$ Craniomaxillofac Surg, 2005, 33, 79.

[30] J. L. Drury, and D. J. Mooney, "Hydrogels for tissue engineering: scaffold design variables and applications", Biomaterials, 2003, 24, 4337.

[31] N. Saito, T. Okada, H. Horiuchi, N. Murakami, J. Takahashi, M. Nawata, et al., "A biodegradable polymer as a cytokine delivery system for inducing bone formation", Nat Biotechnol, 2001, 19, 332.

[32] G. Laverty, S. P. Gorman, and B. F. Gilmore, "Antimicrobial peptide incorporated poly(2-hydroxyethyl methacrylate) hydrogels for the prevention of Staphylococcus epidermidis-associated biomaterial infections", J Biomed Mater Res A, 2012, 100, 1803.

[33] R. Ahmed, S. Fadl-Allah, N. El-Bagoury, and S. Gad El-Rab, "Improvement of corrosion resistance and antibacterial effect of NiTi orthopedic materials by chitosan and gold nanoparticles", App Surf Sci, 2014, 292, 390.

[34] C. A. Engh, J. D. Bobyn, and A. H. Glassman, "Porous-coated hip replacement. The factors governing bone ingrowth, stress shielding, and clinical results", $J$ Bone Joint Surg Br, 1987, 69, 45.

[35] T. Albrektsson, and C. Johansson, "Osteoinduction, osteoconduction and osseointegration", Eur Spine J, 2001, 10 Suppl 2, S96.

[36] H. Kawamura, M. J. Dunbar, P. Murray, R. B. Bourne, and C. H. Rorabeck, "The porous coated anatomic total hip replacement. A ten to fourteen-year follow-up 
study of a cementless total hip arthroplasty", J Bone Joint Surg Am, 2001, 83-A, 1333.

[37] N. P. Hailer, S. Lazarinis, K. T. Makela, A. Eskelinen, A. M. Fenstad, G. Hallan, et al., "Hydroxyapatite coating does not improve uncemented stem survival after total hip arthroplasty!", Acta Orthop, 2015, 86, 18.

[38] R. Z. Legeros, "Properties of osteoconductive biomaterials: calcium phosphates", Clin Orthop Relat Res, 2002, 81.

[39] G. A. Fielding, M. Roy, A. Bandyopadhyay, and S. Bose, "Antibacterial and biological characteristics of silver containing and strontium doped plasma sprayed hydroxyapatite coatings", Acta Biomater, 2012, 8, 3144.

[40] W. Chen, S. Oh, A. P. Ong, N. Oh, Y. Liu, H. S. Courtney, et al., "Antibacterial and osteogenic properties of silver-containing hydroxyapatite coatings produced using a sol gel process", J Biomed Mater Res A, 2007, 82, 899.

[41] M. Kazemzadeh-Narbat, S. Noordin, B. A. Masri, D. S. Garbuz, C. P. Duncan, R. E. Hancock, et al., "Drug release and bone growth studies of antimicrobial peptide-loaded calcium phosphate coating on titanium", J Biomed Mater Res B Appl Biomater, 2012, 100, 1344.

[42] C. J. Pan, Y. X. Dong, Y. Y. Zhang, Y. D. Nie, C. H. Zhao, and Y. L. Wang, "Enhancing the antibacterial activity of biomimetic HA coatings by incorporation of norvancomycin", J Orthop Sci, 2011, 16, 105.

[43] V. Uskokovic, C. Hoover, M. Vukomanovic, D. P. Uskokovic, and T. A. Desai, "Osteogenic and antimicrobial nanoparticulate calcium phosphate and poly-(D,Llactide-co-glycolide) powders for the treatment of osteomyelitis", Mater Sci Eng C Mater Biol Appl, 2013, 33, 3362.

[44] N. Harrison, P. E. Mchugh, W. Curtin, and P. Mc Donnell, "Micromotion and friction evaluation of a novel surface architecture for improved primary fixation of cementless orthopaedic implants", J Mech Behav Biomed Mater, 2013, 21, 37.

[45] N. Harrison, J. R. Field, F. Quondamatteo, W. Curtin, P. E. Mchugh, and P. Mc Donnell, "Preclinical trial of a novel surface architecture for improved primary fixation of cementless orthopaedic implants", Clin Biomech (Bristol, Avon), 2014, $29,861$.

[46] G. Lan, M. Li, Y. Tan, L. Li, X. Yang, L. Ma, et al., "Promoting bone mesenchymal stem cells and inhibiting bacterial adhesion of acid-etched nanostructured titanium by ultraviolet functionalization", J Mater Sci Technol, 2015, 31, 182.

[47] Y. Huang, G. Zha, Q. Luo, J. Zhang, F. Zhang, X. Li, et al., "The construction of hierarchical structure on Ti substrate with superior osteogenic activity and intrinsic antibacterial capability", Sci Rep, 2014, 4, 6172.

[48] Q. Luo, Y. Huang, G. Zha, Y. Chen, X. Deng, K. Zhang, et al., "Topographydependent antibacterial, osteogenic, and anti-aging properties of pure titanium", $J$ Mater Chem B, 2015, 3, 784.

[49] J. R. Jones, "Review of bioactive glass: from Hench to hybrids", Acta Biomater, 2013, 9, 4457.

[50] L. L. Hench, "The story of Bioglass", J Mater Sci Mater Med, 2006, 17, 967. 
[51] F. Ordikhani, and A. Simchi, "Long-term antibiotic delivery by chitosan-based composite coatings with bone regenerative potential", App Surf Sci, 2014, 317, 56.

[52] K. D. Patel, A. El-Fiqi, H. Lee, R. K. Singh, D. Kim, H. Lee, et al., "Chitosannanobioactive glass elecrophoretic coatings with bone regenerative and drug delivering potential", J Mater Chem, 2012, 22, 24945.

[53] A. Bandyopadhyay, S. Bose, and S. Das, "3D printing of biomaterials", MRS Bulletin, 2015, 40, 108.

[54] M. Regis, E. Marin, L. Fedrizzi, and M. Pressacco, "Additive manufacturing of Trabecular Titanium orthopedic implants", MRS Bulletin, 2015, 40, 137.

[55] S. Tarafder, N. M. Davies, A. Bandyopadhyay, and S. Bose, "3D printed tricalcium phosphate scaffolds: Effect of $\mathrm{SrO}$ and $\mathrm{MgO}$ doping on osteogenesis in a rat distal femoral defect model", Biomater Sci, 2013, 1, 1250.

[56] S. Tarafder, V. K. Balla, N. M. Davies, A. Bandyopadhyay, and S. Bose, "Microwave-sintered 3D printed tricalcium phosphate scaffolds for bone tissue engineering", J Tissue Eng Regen Med, 2013, 7, 631.

[57] S. Maleksaeedi, J. K. Wang, A. El-Hajje, L. Harb, V. Guneta, Z. He, et al., "Toward 3D printed bioactive titanium scaffolds with bimodal pore size distribution for bone ingrowth", Procedia CIRP, 2013, 5, 158.

[58] J. K. Bronk, B. H. Russell, J. J. Rivera, R. Pasqualini, W. Arap, M. Hook, et al., "A multifunctional streptococcal collagen-mimetic protein coating prevents bacterial adhesion and promotes osteoid formation on titanium", Acta Biomater, 2014, 10, 3354.

[59] J. Tu, M. Yu, Y. Lu, K. Cheng, W. Weng, J. Lin, et al., "Preparation and antibiotic drug release of mineralized collagen coatings on titanium", J Mater Sci Mater Med, 2012, 23, 2413.

[60] F. Zhang, Z. Zhang, X. Zhu, E. T. Kang, and K. G. Neoh, "Silk-functionalized titanium surfaces for enhancing osteoblast functions and reducing bacterial adhesion", Biomaterials, 2008, 29, 4751.

[61] P. C. Bessa, M. Casal, and R. L. Reis, "Bone morphogenetic proteins in tissue engineering: the road from laboratory to clinic, part II (BMP delivery)", J Tissue Eng Regen Med, 2008, 2, 81.

[62] Z. Shi, K. G. Neoh, E. T. Kang, C. K. Poh, and W. Wang, "Surface functionalization of titanium with carboxymethyl chitosan and immobilized bone morphogenetic protein-2 for enhanced osseointegration", Biomacromolecules, 2009, 10, 1603.

[63] D. W. Lee, Y. P. Yun, K. Park, and S. E. Kim, "Gentamicin and bone morphogenic protein-2 (BMP-2)-delivering heparinized-titanium implant with enhanced antibacterial activity and osteointegration", Bone, 2012, 50, 974.

[64] Z. J. Liu, Y. Zhuge, and O. C. Velazquez, "Trafficking and differentiation of mesenchymal stem cells", J Cell Biochem, 2009, 106, 984.

[65] T. Kitaori, H. Ito, E. M. Schwarz, R. Tsutsumi, H. Yoshitomi, S. Oishi, et al., "Stromal cell-derived factor 1/CXCR4 signaling is critical for the recruitment of mesenchymal stem cells to the fracture site during skeletal repair in a mouse model", Arthritis Rheum, 2009, 60, 813. 
[66] A. Kortesidis, A. Zannettino, S. Isenmann, S. Shi, T. Lapidot, and S. Gronthos, "Stromal-derived factor-1 promotes the growth, survival, and development of human bone marrow stromal stem cells", Blood, 2005, 105, 3793.

[67] E. L. Fong, C. K. Chan, and S. B. Goodman, "Stem cell homing in musculoskeletal injury", Biomaterials, 2011, 32, 395.

[68] S. Zwingenberger, Z. Yao, A. Jacobi, C. Vater, R. D. Valladares, C. Li, et al., "Enhancement of BMP-2 induced bone regeneration by SDF-1alpha mediated stem cell recruitment", Tissue Eng Part A, 2014, 20, 810.

[69] K. Higashino, M. Viggeswarapu, M. Bargouti, H. Liu, L. Titus, and S. D. Boden, "Stromal cell-derived factor-1 potentiates bone morphogenetic protein-2 induced bone formation", Tissue Eng Part A, 2011, 17, 523.

[70] H. D. Hwang, J. T. Lee, J. T. Koh, H. M. Jung, H. J. Lee, and T. G. Kwon, "Sequential Treatment with SDF-1 and BMP-2 Potentiates Bone Formation in Calvarial Defects", Tissue Eng Part A, 2015, 21, 2125.

[71] H. Nagase, M. Miyamasu, M. Yamaguchi, M. Imanishi, N. H. Tsuno, K. Matsushima, et al., "Cytokine-mediated regulation of CXCR4 expression in human neutrophils", J Leukoc Biol, 2002, 71, 711.

[72] S. Oussedik, K. Gould, I. Stockley, and F. S. Haddad, "Defining peri-prosthetic infection: do we have a workable gold standard?", J Bone Joint Surg Br, 2012, 94, 1455.

[73] S. M. Jafari, C. Coyle, S. M. Mortazavi, P. F. Sharkey, and J. Parvizi, "Revision hip arthroplasty: infection is the most common cause of failure", Clin Orthop Relat Res, 2010, 468, 2046.

[74] D. H. Le, S. B. Goodman, W. J. Maloney, and J. I. Huddleston, "Current modes of failure in TKA: infection, instability, and stiffness predominate", Clin Orthop Relat Res, 2014, 472, 2197.

[75] R. Kargupta, S. Bok, C. M. Darr, B. D. Crist, K. Gangopadhyay, S. Gangopadhyay, et al., "Coatings and surface modifications imparting antimicrobial activity to orthopedic implants", Wiley Interdiscip Rev Nanomed Nanobiotechnol, 2014, 6, 475.

[76] W. Zimmerli, and P. E. Ochsner, "Management of infection associated with prosthetic joints", Infection, 2003, 31, 99.

[77] M. Emmerson, "A microbiologist's view of factors contributing to infection", New Horiz, 1998, 6, S3.

[78] K. G. Neoh, X. Hu, D. Zheng, and E. T. Kang, "Balancing osteoblast functions and bacterial adhesion on functionalized titanium surfaces", Biomaterials, 2012, 33, 2813.

[79] P. Kingshott, J. Wei, D. Bagge-Ravn, N. Gadegaard, and L. Gram, "Covalent attachment of poly(ethylene glycol) to surfaces, critical for reducing bacterial adhesion", Langmuir, 2003, 19, 6912.

[80] L. G. Harris, S. Tosatti, M. Wieland, M. Textor, and R. G. Richards, "Staphylococcus aureus adhesion to titanium oxide surfaces coated with nonfunctionalized and peptide-functionalized poly(L-lysine)-grafted-poly(ethylene glycol) copolymers", Biomaterials, 2004, 25, 4135.

[81] F. Yang, C. G. Williams, D. A. Wang, H. Lee, P. N. Manson, and J. Elisseeff, "The effect of incorporating RGD adhesive peptide in polyethylene glycol 
diacrylate hydrogel on osteogenesis of bone marrow stromal cells", Biomaterials, 2005, 26, 5991.

[82] Z. Shi, K. G. Neoh, E. T. Kang, C. Poh, and W. Wang, "Titanium with surfacegrafted dextran and immobilized bone morphogenetic protein-2 for inhibition of bacterial adhesion and enhancement of osteoblast functions", Tissue Eng Part A, 2009, 15, 417.

[83] H.-A. Klock, and J. Genzer, "Expanding the polymer mechanochemistry toolbox through surface-initiated polymerization", ACS Macro Lett, 2015, 4, 636.

[84] K. C. Popat, L. Leoni, C. A. Grimes, and T. A. Desai, "Influence of engineered titania nanotubular surfaces on bone cells", Biomaterials, 2007, 28, 3188.

[85] K. Das, S. Bose, A. Bandyopadhyay, B. Karandikar, and B. L. Gibbins, "Surface coatings for improvement of bone cell materials and antimicrobial activities of $\mathrm{Ti}$ implants", J Biomed Mater Res B Appl Biomater, 2008, 87, 455.

[86] Z. Peng, J. Ni, K. Zheng, Y. Shen, X. Wang, G. He, et al., "Dual effects and mechanism of $\mathrm{TiO} 2$ nanotube arrays in reducing bacterial colonization and enhancing C3H10T1/2 cell adhesion", Int J Nanomedicine, 2013, 8, 3093.

[87] I. Izquierdo-Barba, J. M. Garcia-Martin, R. Alvarez, A. Palmero, J. Esteban, C. Perez-Jorge, et al., "Nanocolumnar coatings with selective behavior towards osteoblast and Staphylococcus aureus proliferation", Acta Biomater, 2015, 15, 20.

[88] Y. Li, W. Xiong, C. Zhang, B. Gao, H. Guan, H. Cheng, et al., "Enhanced osseointegration and antibacterial action of zinc-loaded titania-nanotube-coated titanium substrates: in vitro and in vivo studies", J Biomed Mater Res A, 2014, $102,3939$.

[89] L. Zhao, H. Wang, K. Huo, L. Cui, W. Zhang, H. Ni, et al., "Antibacterial nanostructured titania coating incorporated with silver nanoparticles", Biomaterials, 2011, 32, 5706.

[90] R. Hang, A. Gao, X. Huang, X. Wang, X. Zhang, L. Qin, et al., "Antibacterial activity and cytocompatibility of Cu-Ti-O nanotubes", J Biomed Mater Res A, 2014, 102, 1850.

[91] S. Eckhardt, P. S. Brunetto, J. Gagnon, M. Priebe, B. Giese, and K. M. Fromm, "Nanobio silver: its interactions with peptides and bacteria, and its uses in medicine", Chem Rev, 2013, 113, 4708.

[92] H. Cao, X. Liu, F. Meng, and P. K. Chu, "Biological actions of silver nanoparticles embedded in titanium controlled by micro-galvanic effects", Biomaterials, 2011, 32, 693.

[93] H. Cao, T. Cui, G. Jin, and X. Liu, "Cellular responses to titanium successively treated by magnesium and silver PIII\&D", Surf Coat Tech, 2014, 256, 9.

[94] H. Cao, Y. Qiao, X. Liu, T. Lu, T. Cui, F. Meng, et al., "Electron storage mediated dark antibacterial action of bound silver nanoparticles: smaller is not always better", Acta Biomater, 2013, 9, 5100.

[95] S. Qiao, H. Cao, X. Zhao, H. Lo, L. Zhuang, Y. Gu, et al., "Ag-plasma modification enhances bone apposition around titanium dental implants: an animal study in Labrador dogs", Int J Nanomedicine, 2015, 10, 653.

[96] G. Jin, H. Qin, H. Cao, S. Qian, Y. Zhao, X. Peng, et al., "Synergistic effects of dual $\mathrm{Zn} / \mathrm{Ag}$ ion implantation in osteogenic activity and antibacterial ability of titanium", Biomaterials, 2014, 35, 7699. 
[97] R. Huang, Y. Han, and S. Lu, "Enhanced osteoblast functions and bactericidal effect of $\mathrm{Ca}$ and $\mathrm{Ag}$ dual-ion implanted surface layers on nanograined titanium alloys", J Mater Chem B, 2014, 2, 4531.

[98] A. Di Martino, M. Sittinger, and M. V. Risbud, "Chitosan: a versatile biopolymer for orthopaedic tissue-engineering", Biomaterials, 2005, 26, 5983.

[99] C. Shi, Y. Zhu, X. Ran, M. Wang, Y. Su, and T. Cheng, "Therapeutic potential of chitosan and its derivatives in regenerative medicine", J Surg Res, 2006, 133, 185.

[100] M. Dash, F. Chiellini, R. M. Ottenbrite, and E. Chiellini, "Chitosan - a versatile semi-synthetic polymer in biomedical applications", Prog Polym Sci, 2011, 36, 981.

[101] E. I. Rabea, M. E. Badawy, C. V. Stevens, G. Smagghe, and W. Steurbaut, "Chitosan as antimicrobial agent: applications and mode of action", Biomacromolecules, 2003, 4, 1457.

[102] Z. Shi, K. G. Neoh, E. T. Kang, C. Poh, and W. Wang, "Bacterial adhesion and osteoblast function on titanium with surface-grafted chitosan and immobilized RGD peptide", J Biomed Mater Res A, 2008, 86, 865.

[103] P. H. Chua, K. G. Neoh, E. T. Kang, and W. Wang, "Surface functionalization of titanium with hyaluronic acid/chitosan polyelectrolyte multilayers and RGD for promoting osteoblast functions and inhibiting bacterial adhesion", Biomaterials, 2008, 29, 1412 .

[104] D. Zheng, K. G. Neoh, Z. Shi, and E. T. Kang, "Assessment of stability of surface anchors for antibacterial coatings and immobilized growth factors on titanium", $J$ Colloid Interface Sci, 2013, 406, 238.

[105] F. Ordikhani, E. Tamjid, and A. Simchi, "Characterization and antibacterial performance of electrodeposited chitosan-vancomycin composite coatings for prevention of implant-associated infections", Mater Sci Eng C Mater Biol Appl, 2014, 41, 240.

[106] M. Mattioli-Belmonte, S. Cometa, C. Ferretti, R. Iatta, A. Trapani, E. Ceci, et al., "Characterization and cytocompatibility of an antibiotic/chitosan/cyclodextrins nanocoating on titanium implants", Carbohydr Polym, 2014, 110, 173.

[107] L. Zhao, Y. Hu, D. Xu, and K. Cai, "Surface functionalization of titanium substrates with chitosan-lauric acid conjugate to enhance osteoblasts functions and inhibit bacteria adhesion", Colloids Surf, B, 2014, 119, 115.

[108] J. L. Del Pozo, and R. Patel, "Clinical practice. Infection associated with prosthetic joints", N Engl J Med, 2009, 361, 787.

[109] H. Van De Belt, D. Neut, W. Schenk, J. R. Van Horn, H. C. Van Der Mei, and H. J. Busscher, "Infection of orthopedic implants and the use of antibiotic-loaded bone cements. A review", Acta Orthop Scand, 2001, 72, 557.

[110] W. M. Dunne Jr., "Bacterial adhesion: seen any good biofilms lately?", Clin Microbiol Rev, 2002, 15, 155.

[111] E. M. Hetrick, and M. H. Schoenfisch, "Reducing implant-related infections: active release strategies", Chem Soc Rev, 2006, 35, 780.

[112] J. W. Costerton, K. J. Cheng, G. G. Geesey, T. I. Ladd, J. C. Nickel, M. Dasgupta, et al., "Bacterial biofilms in nature and disease", Annu Rev Microbiol, 1987, 41, 435. 
[113] V. Antoci, Jr., C. S. Adams, J. Parvizi, P. Ducheyne, I. M. Shapiro, and N. J. Hickok, "Covalently attached vancomycin provides a nanoscale antibacterial surface", Clin Orthop Relat Res, 2007, 461, 81.

[114] V. Antoci, Jr., C. S. Adams, J. Parvizi, H. M. Davidson, R. J. Composto, T. A. Freeman, et al., "The inhibition of Staphylococcus epidermidis biofilm formation by vancomycin-modified titanium alloy and implications for the treatment of periprosthetic infection", Biomaterials, 2008, 29, 4684.

[115] N. J. Hickok, and I. M. Shapiro, "Immobilized antibiotics to prevent orthopaedic implant infections", Adv Drug Deliv Rev, 2012, 64, 1165.

[116] C. Marambio-Jones, and E. M. V. Hoek, "A review of the antibacterial effects of silver nanomaterials and potential implications for human health and the environment", J Nanopart Res, 2010, 12, 1531.

[117] P. V. Asharani, G. Low Kah Mun, M. P. Hande, and S. Valiyaveettil, "Cytotoxicity and genotoxicity of silver nanoparticles in human cells", ACS Nano, 2009, 3, 279.

[118] Y. Liu, Z. Zheng, J. N. Zara, C. Hsu, D. E. Soofer, K. S. Lee, et al., "The antimicrobial and osteoinductive properties of silver nanoparticle/poly (DL-lacticco-glycolic acid)-coated stainless steel", Biomaterials, 2012, 33, 8745.

[119] A. Gao, R. Hang, X. Huang, L. Zhao, X. Zhang, L. Wang, et al., "The effects of titania nanotubes with embedded silver oxide nanoparticles on bacteria and osteoblasts", Biomaterials, 2014, 35, 4223.

[120] A. Agarwal, T. L. Weis, M. J. Schurr, N. G. Faith, C. J. Czuprynski, J. F. Mcanulty, et al., "Surfaces modified with nanometer-thick silver-impregnated polymeric films that kill bacteria but support growth of mammalian cells", Biomaterials, 2010, 31, 680.

[121] C. M. Tilmaciu, M. Mathieu, J. P. Lavigne, K. Toupet, G. Guerrero, A. Ponche, et al., "In vitro and in vivo characterization of antibacterial activity and biocompatibility: a study on silver-containing phosphonate monolayers on titanium", Acta Biomater, 2015, 15, 266.

[122] P. J. Finley, R. Norton, C. Austin, A. Mitchell, S. Zank, and P. Durham, "Unprecedented Silver Resistance in Clinically Isolated Enterobacteriaceae: Major Implications for Burn and Wound Management", Antimicrob Agents Chemother, 2015, 59, 4734.

[123] H. Hu, W. Zhang, Y. Qiao, X. Jiang, X. Liu, and C. Ding, "Antibacterial activity and increased bone marrow stem cell functions of $\mathrm{Zn}$-incorporated $\mathrm{TiO} 2$ coatings on titanium", Acta Biomater, 2012, 8, 904.

[124] K. Huo, X. Zhang, H. Wang, L. Zhao, X. Liu, and P. K. Chu, "Osteogenic activity and antibacterial effects on titanium surfaces modified with Zn-incorporated nanotube arrays", Biomaterials, 2013, 34, 3467.

[125] G. Jin, H. Cao, Y. Qiao, F. Meng, H. Zhu, and X. Liu, "Osteogenic activity and antibacterial effect of zinc ion implanted titanium", Colloids Surf, B, 2014, 117, 158.

[126] M. Tsai, Y. Chang, H. Huang, J. Hsu, Y. Chen, and A. Wu, "Characterization and antibacterial performance of bioactive Ti-Zn-O coatings deposited on titanium implants", Thin Solid Films, 2013, 528, 143. 
[127] S. Svensson, F. Suska, L. Emanuelsson, A. Palmquist, B. Norlindh, M. Trobos, et al., "Osseointegration of titanium with an antimicrobial nanostructured noble metal coating", Nanomedicine, 2013, 9, 1048.

[128] K. C. Popat, M. Eltgroth, T. J. Latempa, C. A. Grimes, and T. A. Desai, "Decreased Staphylococcus epidermis adhesion and increased osteoblast functionality on antibiotic-loaded titania nanotubes", Biomaterials, 2007, 28, 4880.

[129] R. V. Ulijn, N. Bibi, V. Jayawarna, P. D. Thornton, S. J. Todd, R. J. Mart, et al., "Bioresponsive hydrogels", Mater Today, 2007, 10, 40.

[130] D. A. Wang, C. G. Williams, F. Yang, N. Cher, H. Lee, and J. H. Elisseeff, "Bioresponsive phosphoester hydrogels for bone tissue engineering", Tissue Eng, 2005, 11, 201.

[131] N. Singh, A. Karambelkar, L. Gu, K. Lin, J. S. Miller, C. S. Chen, et al., "Bioresponsive mesoporous silica nanoparticles for triggered drug release", J Am Chem Soc, 2011, 133, 19582.

[132] D. Pornpattananangkul, L. Zhang, S. Olson, S. Aryal, M. Obonyo, K. Vecchio, et al., "Bacterial toxin-triggered drug release from gold nanoparticle-stabilized liposomes for the treatment of bacterial infection", J Am Chem Soc, 2011, 133, 4132.

[133] C. D. Fjell, J. A. Hiss, R. E. Hancock, and G. Schneider, "Designing antimicrobial peptides: form follows function", Nat Rev Drug Discov, 2012, 11, 37.

[134] R. E. Hancock, and H. G. Sahl, "Antimicrobial and host-defense peptides as new anti-infective therapeutic strategies", Nat Biotechnol, 2006, 24, 1551.

[135] M. Kazemzadeh-Narbat, J. Kindrachuk, K. Duan, H. Jenssen, R. E. Hancock, and R. Wang, "Antimicrobial peptides on calcium phosphate-coated titanium for the prevention of implant-associated infections", Biomaterials, 2010, 31, 9519.

[136] M. Kazemzadeh-Narbat, B. F. Lai, C. Ding, J. N. Kizhakkedathu, R. E. Hancock, and R. Wang, "Multilayered coating on titanium for controlled release of antimicrobial peptides for the prevention of implant-associated infections", Biomaterials, 2013, 34, 5969.

[137] G. Gao, D. Lange, K. Hilpert, J. Kindrachuk, Y. Zou, J. T. Cheng, et al., "The biocompatibility and biofilm resistance of implant coatings based on hydrophilic polymer brushes conjugated with antimicrobial peptides", Biomaterials, 2011, 32, 3899.

[138] R. Rathinakumar, W. F. Walkenhorst, and W. C. Wimley, "Broad-spectrum antimicrobial peptides by rational combinatorial design and high-throughput screening: the importance of interfacial activity", J Am Chem Soc, 2009, 131, 7609.

[139] W. L. Maloy, and U. P. Kari, "Structure-activity studies on magainins and other host defense peptides", Biopolymers, 1995, 37, 105.

[140] K. Hamamoto, Y. Kida, Y. Zhang, T. Shimizu, and K. Kuwano, "Antimicrobial activity and stability to proteolysis of small linear cationic peptides with D-amino acid substitutions", Microbiol Immunol, 2002, 46, 741.

[141] M. J. Sweet, and D. A. Hume, "Endotoxin signal transduction in macrophages", $J$ Leukoc Biol, 1996, 60, 8. 
[142] J. Pajarinen, E. Jamsen, Y. T. Konttinen, and S. B. Goodman, "Innate immune reactions in septic and aseptic osteolysis around hip implants", J Long Term Eff Med Implants, 2014, 24, 283.

[143] M. Benoit, B. Desnues, and J. L. Mege, "Macrophage polarization in bacterial infections", J Immunol, 2008, 181, 3733.

[144] S. L. Teitelbaum, "Bone resorption by osteoclasts", Science, 2000, 289, 1504.

[145] W. J. Boyle, W. S. Simonet, and D. L. Lacey, "Osteoclast differentiation and activation", Nature, 2003, 423, 337.

[146] E. Jimi, K. Aoki, H. Saito, F. D'acquisto, M. J. May, I. Nakamura, et al., "Selective inhibition of NF-kappa B blocks osteoclastogenesis and prevents inflammatory bone destruction in vivo", Nat Med, 2004, 10, 617.

[147] S. Morony, C. Capparelli, R. Lee, G. Shimamoto, T. Boone, D. L. Lacey, et al., "A chimeric form of osteoprotegerin inhibits hypercalcemia and bone resorption induced by IL-1beta, TNF-alpha, PTH, PTHrP, and 1, 25(OH)2D3", J Bone Miner Res, 1999, 14, 1478.

[148] S. Franz, S. Rammelt, D. Scharnweber, and J. C. Simon, "Immune responses to implants - a review of the implications for the design of immunomodulatory biomaterials", Biomaterials, 2011, 32, 6692.

[149] A. Oberholzer, C. Oberholzer, and L. L. Moldawer, "Sepsis syndromes: understanding the role of innate and acquired immunity", Shock, 2001, 16, 83.

[150] D. M. Bowdish, D. J. Davidson, Y. E. Lau, K. Lee, M. G. Scott, and R. E. Hancock, "Impact of LL-37 on anti-infective immunity", J Leukoc Biol, 2005, 77, 451.

[151] M. G. Scott, E. Dullaghan, N. Mookherjee, N. Glavas, M. Waldbrook, A. Thompson, et al., "An anti-infective peptide that selectively modulates the innate immune response", Nat Biotechnol, 2007, 25, 465.

[152] A. L. Hilchie, K. Wuerth, and R. E. Hancock, "Immune modulation by multifaceted cationic host defense (antimicrobial) peptides", Nat Chem Biol, 2013, 9, 761. 